\section{Kidney/Renal Pathology (including Transplantation)}

1614 Molecular Assessment of Transplant Renal Biopsies and Comparison with Histology

John Aird, Claire Kennedy, Donal Sexton, Jeff Reeve, Philip Halloran, Declan de Freitas, Brendan Doyle. Royal College of Surgeons in Ireland, Dublin, Ireland; Beaumont Hospital, Dublin, Ireland; University of Alberta, Edmonton, AB, Canada. Background: "The Molecular Microscope ${ }^{\otimes} "$ utilises a combined microarray and reference biopsy library to determine a probability score for disease activity, stage and prognosis in renal transplant biopsies. Here we describe an audit of our initial experience using this approach to complement traditional biopsies.

Design: At the time of renal biopsy, an additional core was taken and shipped to the University of Alberta in RNALater. Renal biopsies were reported by 2 renal pathologists using the Banff Classification, without knowledge of the molecular results. "The Molecular Microscope ${ }^{\circledR} "$ assay was performed without clinical or histological information. Both techniques were then compared and ultimately a histo-molecular phenotype was arrived at.

Results: To date we have collected data from 6 patients. A molecular report was returned between 3-6 days after the biopsy depending on day of shipping. Table 1 shows the histological, molecular and clinical diagnoses. In two of the cases which showed rejection the biopsy for histology was sub-optimal and the correlation with the molecular phenotype provided extra evidence on which to base clinical decisions. Table 1. TCMR: T-cell mediated rejection, BKN: BK Nephropathy, ATI: Acute tubular injury, AKI: Acute kidney injury, ABMR: Acute antibody mediated rejection

\begin{tabular}{|l|l|l|l|}
\hline & Histology & Molecular & Clinical \\
\hline 1 & TCMR & TCMR & TCMR \\
\hline 2 & BKN & AKI & BKN \\
\hline 3 & Infarction & AKI & AKI \\
\hline 4 & ATI & AKI & AKI \\
\hline 5 & Mixed rejection & ABMR & ABMR \\
\hline 6 & ATI & AKI & AKI \\
\hline
\end{tabular}

Interestingly, the biopsy which was infarcted was read molecularly as all medulla, based on a lack of podocin expression. It remains to be seen if this genuinely represents medulla or an artefact of the infarction. In this case the molecular phenotype was of acute kidney injury. On this basis no treatment was instigated and the patient's renal function improved spontaneously, supporting the molecular diagnosis. In general, the molecular assay also predicted the histological scores of the biopsies.

Conclusions: The use of molecular techniques is well established in tumour pathology, but remains at an early stage in transplant renal pathology. We have seen in this series that the results generally correlated well with histology and can add value in terms of clinical decision making. It is important that clinical phenotype, histology and molecular techniques are integrated, in order to reach the optimal diagnosis.

\section{Renal Pathology Findings in POEMS Syndrome}

Mariam P Alexander, Lynn D Cornell, Thomas Czeczok. Mayo Clinic, Rochester, MN. Background: Individuals with polyneuropathy, organomegaly, endocrinopathy, monoclonal gammopathy, and skin changes (POEMS) syndrome often have renal dysfunction. Renal biopsy findings in a Caucasian population is not well described. This study aims to describe presentation, rbx findings, \& outcomes in the setting of POEMS syndrome.

Design: The renal pathology database was searched for POEMS syndrome from 20012016. Chart review was performed including clinical presentation, laboratory testing, and follow-up. Pathology was reviewed when available.

Results: Nine biopsies were identified; all were Caucasian. Findings are presented in Table1. There was a male dominance $(\mathrm{n}=7)$. Indications for biopsy included proteinuria $(n=4 ; 2$ with nephrotic range), chronic renal failure $(n=2)$, and acute kidney injury $(\mathrm{n}=2)$. VEGF and IL-6 were elevated in 6 and 5 individuals, respectively. Biopsies showed thrombotic microangiopathy (TM) $(n=8)$, membranous nephropathy $(n=1)$, crescentic and endocapillary proliferative glomerulonephritis with linear $\operatorname{IgA} \lambda(\mathrm{n}=1)$, diabetic glomerulosclerosis with resolving post infectious $\mathrm{GN}(\mathrm{n}=1)$, and immunotactoid glomerulopathy $(\mathrm{n}=1)$. Interstitial fibrosis was mild $(0-30 \%)$. Electron microscopy showed variable endothelial and subendothelial injury, glomerular basement membrane remodeling, and mesangiolysis. Serum creatinine and 24-hour urine proteins did not significantly improve with treatment.

\begin{tabular}{|c|c|c|c|c|c|c|c|}
\hline Case & $\begin{array}{c}\text { Age/ } \\
\text { Gen- } \\
\text { der }\end{array}$ & $\begin{array}{l}\text { Serum } \\
\text { Cre- } \\
\text { atinine } \\
\text { (mg/ } \\
\text { dL) at } \\
\text { Presenta- } \\
\text { tion }\end{array}$ & $\begin{array}{l}24-\text {-hr } \\
\text { Urine } \\
\text { Protein } \\
\text { (g) }\end{array}$ & $\begin{array}{l}\text { Pathologic Di- } \\
\text { agnosis }\end{array}$ & Treatment & $\begin{array}{l}\text { Follow- } \\
\text { Up Cre- } \\
\text { atinine } \\
(\mathrm{mg} / \\
\mathrm{dL})\end{array}$ & $\begin{array}{l}\text { Follow- } \\
\text { Up 24- } \\
\text { hr Urine } \\
\text { Protein } \\
\text { (g) }\end{array}$ \\
\hline 1 & $59 \mathrm{M}$ & 1.5 & 1,112 & TM & SCT & 2.0 & - \\
\hline 2 & $56 \mathrm{~F}$ & 1.5 & 500 & TM & $\begin{array}{c}\text { Sacral Plas- } \\
\text { macytoma } \\
\text { Radiation, } \\
\text { Prednisone }\end{array}$ & 2.8 & 73 \\
\hline 3 & $72 \mathrm{M}$ & 2.3 & 123 & TM & $\begin{array}{c}\text { Bortezomib, } \\
\text { Cyclophospha- } \\
\text { mide, Dexa- } \\
\text { methasone }\end{array}$ & 2.4 & 173 \\
\hline 4 & $57 \mathrm{M}$ & 1.1 & 16,200 & $\begin{array}{l}\text { Membranous } \\
\text { Nephropathy }\end{array}$ & $\begin{array}{l}\text { SCT, Revlimid, } \\
\text { Dexametha- } \\
\text { zone }\end{array}$ & 1.7 & 102 \\
\hline 5 & $41 \mathrm{M}$ & 1.2 & 7,500 & $\begin{array}{l}\text { TM, Crescentic } \\
\text { and Endocapil- } \\
\text { lary Prolifera- } \\
\text { tive Glomeru- } \\
\text { lonephritis with } \\
\text { Linear IgA } \lambda\end{array}$ & $\begin{array}{l}\text { SCT, Ixazomib, } \\
\text { Dexametha- } \\
\text { sone. }\end{array}$ & 1.1 & 324 \\
\hline 6 & $51 \mathrm{M}$ & 1.9 & 72 & $\begin{array}{l}\text { Diabetic Ne- } \\
\text { phropathy, TM }\end{array}$ & SCT & 2.0 & - \\
\hline 7 & $69 \mathrm{M}$ & 2.9 & 257 & TM & - & - & - \\
\hline 8 & $69 \mathrm{M}$ & 2.2 & 146 & TM & $\begin{array}{c}\text { Rituximab, } \\
\begin{array}{c}\text { Cyclophospha- } \\
\text { mide, Predni- } \\
\text { sone }\end{array} \\
\end{array}$ & 2.1 & 126 \\
\hline 9 & $64 \mathrm{~F}$ & - & - & $\begin{array}{l}\text { Immunotactoid } \\
\text { Glomerulopa- } \\
\text { thy, TM }\end{array}$ & - & - & - \\
\hline
\end{tabular}

Conclusions: This is the first series of renal biopsies in individuals with POEMS syndrome in a caucasian population. While thrombotic microangiopathy is the most common finding, immune complex glomerulonephritis may occur. Elevated VEGF and IL-6 likely contribute to pathology.

1616 Chimerism in Allograft Kidney Tumors in Transplanted Patients Hussein Alnajar, Sahr Syed, David Cimbaluk, Lela Buckingham, Paolo Gattuso. Rush University Medical Center, Chicago, IL.

Background: In renal transplant patients, tumors more commonly arise in native endstage kidney, while allograft tumors are extremely rare. To date, genetic studies to trace the origin of these tumors have been performed in just a few cases.

Design: We performed a retrospective analysis of allograft biopsies and transplant nephrectomies at our institution from 1993-2016, to elucidate donor versus recipient origin of native kidney tumors in renal transplant patients.

Results: We identified a total of 4521 specimens including 189 allograft nephrectomies and 4332 allograft renal biopsies. Nine cases of renal allograft tumors were identified ( $0.2 \%$ of allograft specimens). Of these, renal cortical adenoma was the most common tumor (4 cases, $44 \%$ ), followed by urothelial cell carcinoma (UCC) of the renal pelvis ( 2 cases, 22\%), post-transplant lymphoproliferative disorder ( 2 cases, $22 \%$ ) and oncocytoma ( 1 case, $12 \%$ ). The mean age was 50 years (range $28-73$ ). Mean interval between the time of renal transplant and the development of neoplasm was 102 months (range $1-255$ months).

Most solid-organ or hematopoietic stem-cell transplantations are allogeneic and chimerism studies can distinguish donor and recipient tissue origin. DNA extracts from eight tumors and benign tissue from the recipient were submitted for chimerism analysis. Donor reference tissue was not available. Six cases showed different allelic profiles in donor and recipient DNA. Matching confirmed the recipient origin of tumor cells in the other two cases.

Conclusions: Allograft renal tumors are extremely rare $(0.2 \%$ of cases). Renal cortical adenoma was the most common neoplasm of the allograft renal tumors. Tumor cells developed in the allograft kidney may arise from the donor's kidney, or may originate from a recipient origin. The possibility of development of malignancies in allograft kidney may actually have occurred prior to transplantation. A de novo origin can be assumed if the tumor occurs later than 6 months after the transplant. Only two patients developed tumor earlier than six months; however, the possibility of an occult slow growing tumor in allograft cannot be ruled out.

Renal or bone marrow stem cells migrating to the allograft kidney with subsequent malignant transformation was proposed in tumor with recipient origin. A possibility of metastasis from the native kidney was excluded by imaging studies and follow up. Once the diagnosis of tumor in the allograft kidney is made, it is crucial to obtain information about tumor cell origin, especially to assess potential transmission of a neoplasm to other recipients from the same donor. 
1617 Donor-Derived Metastatic Melanoma Transmitted Through Kidney Allograft

Shaymaa AL-L Ashi, Andrei Plagov, Alden Doyle, Suzanne M Boyle, Suganthi Soundararajan. Drexel University, Philadephia, PA.

Background: Transmission of melanoma from organ donors is rare and has been reported. Diagnosis of de nova melanoma can be challenging in a renal allograft patient. Immunohistochemical studies, cytogenetic analysis, donor history and tracking of the outcome of organ recipients from the same donor can aid in the diagnosis of donorderived metastatic melanoma.

Design: A 57 year old male presented with pain around the allograft and increasing creatinine, 7 months after deceased-donor kidney transplantation. His past medical history was significant for chronic glomerulonephritis following kidney donation and prostatic adenocarcinoma (Gleason score: 8, pT3a N0), status post treatment. Allograft kidney biopsy was negative for rejection. Imaging studies revealed multiple nodules in both lungs and liver, mild hydronephrosis and normal resistive indices in the allograft. Results: Biopsy of the lung nodules revealed poorly differentiated spindle cell proliferation which expressed S100, MART-1 and HMB-45 consistent with metastatic melanoma. BRAF-V600E (C.1799T >A) mutation was detected. Prostate markers were negative. Immunosuppression was stopped and allograft nephrectomy was performed. Multiple bulky, well delineated masses with abundant necrosis were present in the hilum, subcapsular region, capsule and perirenal adipose tissue, with histomorphology similar to the lung tumor (pTX NX M1). Extensive lymphovascular invasion was present. No tumor infiltrating lymphocytes were noted. The surrounding kidney had no evidence of rejection. The patient responded well to BRAF/ MEK inhibition and checkpoin inhibitors. One year after the presentation, the patient is alive, dialysis-dependent and has evidence of tumor regression.

At a different institution, the female recipient of the liver and the other kidney from the same donor, developed metastatic melanoma around the same period. Cytogenetic testing detected $\mathrm{XY}$ chromosomes in the tumor cells. The donor was a young male who died of intracerebral hemorrhage.

Conclusions: Immunohistochemical studies and cytogenetic analysis, assisted in the diagnosis of metastatic melanoma of donor origin, in a kidney transplant recipient from a deceased donor. Intracranial hemorrhage in a young patient can be caused by an undiagnosed melanoma.

1618 Sox9 Potentially Distinguishes Focal Segmental Glomerulosclerosis from Minimal Change Disease

Beth L Braunhut, Vincent M Cracolici, Kammi J Henriksen, Mariana M Cajaiba, Anthony Chang. The University of Chicago, Chicago, IL; Lurie Children's Hospital, Chicago, IL.

Background: SYR-related HMG box 9 (SOX9) has been associated with up-regulation of genes related to fibrosis, namely COL2A2, COMP1, and COL1. We hypothesized that Sox 9 expression may be increased in glomerular diseases with the potential for glomerular and interstitial fibrosis and sought to determine the significance of Sox9 expression.

Design: We searched the pathology archives at our institution from 2013-2015 for kidney biopsies with the following diagnoses: minimal change disease (MCD, 14), IgM nephropathy (IgMN, 7), and focal segmental glomerulosclerosis (FSGS, 16) including the tip (3), cellular (1), perihilar (2), and collapsing (1) variants. Controls included membranous nephropathy (MN, 3), diabetic nephropathy (mild and advanced, 4 cases each), IgA nephropathy (IgAN, 5), and chronic transplant glomerulopathy (TG, 5). Sox9 immunohistochemistry was performed on formalin-fixed, paraffin-embedded tissue samples and nuclear staining of glomerular, tubular, and interstitial nuclei was assessed. When positive, the staining was scored as follows: $1+(1-25 \%), 2+(26-50 \%)$, and $3+(>50 \%)$

Results: FSGS demonstrated Sox 9 expression in tubular epithelial nuclei 8 of 16 cases, $50 \%$ ) and/or parietal epithelial nuclei (5 of 16,31\%). Parietal epithelial cell staining was diffuse (3+) in all positive cases. Tubular cell expression was predominantly in distal nephron segments and varied in the 8 positive cases: $4(50 \%)$ with $1+$ staining, $1(13 \%)$ with $2+$ staining and 3 cases $(38 \%)$ with $3+$ staining. There was no significant expression of Sox 9 in MCD or IgMN with the exception of one MCD case that showed both parietal and tubular epithelial cell staining (1+ for each). There was also no significant Sox 9 staining in $\mathrm{MN}(0$ of $3,0 \%)$ or advanced $\mathrm{DN}(0$ of $4,0 \%)$. There was Sox9 tubular expression in $3(75 \%)$ mild DN cases and $2(40 \%)$ IgAN cases. Chronic TG showed expression in parietal epithelial cells in $1(20 \%)$ case. There seemed to be some association between Sox 9 expression and extent of interstitial fibrosis and tubular atrophy with more staining in atrophic tubules.

Conclusions: Sox9 expression may be useful in differentiating MCD from FSGS, especially in limited biopsy samples. Based on these expression patterns, the potential role of parietal and tubular epithelial cells in glomerular and tubulointerstitial fibrosis warrants additional investigation.

\section{9}

De Novo Pauci-Immune Glomerulonephritis in the Renal Allograft

Alessia Buglioni, Mary E Fidler, Sanjeev Sethi, Samih H Nasr, Loren P Herrera Hernandez, Fernando Cosio, Lynn D Cornell. Mayo Clinic, Rochester, MN.

Background: Pauci-immune glomerulonephritis (GN) in the native kidney presents with acute kidney injury, hematuria, and proteinuria, and is usually due to anti-neutrophil cytoplasmic antibodies (ANCA). On biopsy, glomeruli show necrosis and crescents, with a pauci-immune pattern by immunofluorescence. Rarely, kidney transplants can show this pattern as de novo disease.

Design: A retrospective analysis with clinicopathological correlation was performed in cases of de novo pauci-immune GN on kidney transplant biopsies diagnosed between Jan 2009 and Jan 2016.
Results: Eight patients (3 M, 5 F) were identified that showed post-transplant de novo pauci-immune GN on biopsy, accounting for $<0.001 \%$ of all transplant biopsies. The mean age was 52 years (range 26-78). The native kidney diseases were lupus nephritis $(\mathrm{n}=3)$, polycystic kidney disease $(\mathrm{n}=2)$, diabetes $(\mathrm{n}=1)$, interstitial nephritis $(\mathrm{n}=1)$, and focal segmental glomerulosclerosis $(\mathrm{n}=1)$. Three patients had protocol biopsies and 5 had biopsies for elevated serum creatinine (SCr). The mean SCr at biopsy was $3.3 \mathrm{mg}$ $\mathrm{dl}$ (range 1.4-7.9). All patients had hematuria and 7 had mild proteinuria at the time of biopsy. The mean biopsy time post-transplant was 33 months (range 4-96); 3 occurred within the first 13 months post-transplant. Five patients were on maintenance triple immunosuppression with tacrolimus, MMF, and prednisone, 1 was on a steroid-free protocol, 2 were on tacrolimus and prednisone. There was no evidence of systemic vasculitis in any patient.

All biopsies showed focal necrotizing or crescentic GN (mean 19\% glomeruli involved, range 2-36), 7 showed mild and 1 showed moderate interstitial fibrosis. None showed necrotizing arteritis. Immunofluorescence and electron microscopy showed no evidence of immune complex GN in all biopsies. ANCA (anti-MPO and -PR3) results were available for 7 patients and were negative in all but one patient.

Clinical follow-up information was available in 7 patients. Two patients lost their grafts 1 and 3 years after the biopsy showing pauci-immune GN. In the other patients, the mean most recent SCr was $1.7 \mathrm{mg} / \mathrm{dl}$, range 1.4-2.3, average 38 months (range 7-78) after the biopsy. Three patients died at 36, 21 and 7 months after biopsy diagnosis. Two patients had repeat biopsies that showed continued pauci-immune GN.

Conclusions: This is the first series describing post-transplant de novo pauci-immune $\mathrm{GN}$, which appears to be a unique pathologic entity that may occur early or late posttransplant and is usually not associated with ANCA or systemic vasculitis. The degree of crescent formation and renal impairment are milder than those of pauci-immune GN in the native kidney.

\section{Optimal Tissue Preparation of Donor Kidney Biopsies for Rapid} Digital Pathology Interpretation

Jie Chen, Satoru Kudose, Joseph Gaut. Washington University in St. Louis, St. Louis, MO.

Background: Preimplantation frozen wedge renal biopsies are often used by transplant centers to make determinations regarding organ acceptance. A recent study reported by the Banff working group indicated superiority of frozen wedge biopsies compared with frozen core biopsies but reported only fair reproducibility for a number of pathologic parameters. Rapid formalin fixation and processing of tissue has the potential to improve reproducibility and evaluation of preimplantation kidney biopsies.

Design: Rapid interpretation of preimplantation kidney biopsies using digital pathology is routinely performed at our center. Two organ procurement organizations refer these biopsies using different techniques: frozen wedge biopsy and rapid formalin fixation core biopsy. All rapid interpretations are performed by general surgical pathologists. Permanent glass slides are reviewed by a renal pathologist. The most recent consecutive 50 cases of frozen wedge biopsies and 50 cases of rapid formalin fixed core biopsies were evaluated. The discrepancy rates (DR) between the digital intraoperative diagnoses and the final glass slide diagnoses were compared for the following parameters: number of glomeruli, number of globally sclerotic glomeruli, interstitial fibrosis, acute tubular injury, arterial intimal fibrosis, and arteriolar hyalinosis. Fisher's exact tests were performed to compare the discrepancy rates.

Results: Rapid formalin fixation of core biopsies yielded more accurate detection of arterial intimal thickening than wedge biopsies ( $\mathrm{DR}=10 \%$ vs $28 \%$;

$\mathrm{P}=0.0395)$ and tended to more accurate detection of interstitial fibrosis ( $\mathrm{DR}=8 \%$ vs $20 \%$ ) and arteriolar hyalinosis ( $\mathrm{DR}=20 \%$ vs $32 \%$ ), although these were not statistically significant. The discrepancy rates for number of glomeruli $(\mathrm{DR}=16 \% \mathrm{vs} 12 \%)$, number of globally sclerotic glomeruli ( $\mathrm{DR}=12 \%$ vs $12 \%$ ), and acute tubular injury ( $\mathrm{DR}=28 \%$ vs $32 \%$ ) were fairly similar using both needle biopsies and wedge biopsies.

Conclusions: Our results indicate that general pathologist interpretation of rapid formalin fixed core kidney biopsies using digital pathology has a lower rate of discrepancy with permanent section review by a renal pathologist compared to frozen wedge biopsies. Further studies evaluating the reproducibility between pathologists using these two techniques are indicated.

\section{Spectrum of IgA-Associated Glomerular Lesions in Patients} with Liver Disease

Vincent M Cracolici, Beth L Braunhut, Anthony Chang, Kammi J Henriksen. University of Chicago Medical Center, Chicago, IL.

Background: IgA nephropathy (IgAN) is the most common glomerulonephritis (GN) identified in renal biopsies worldwide, and shows considerable heterogeneity of clinical behavior and pathologic expression. IgAN occurs as a primary disease or secondary to underlying conditions. Despite the well-known association between liver disease and IgA nephropathy, the incidence and pathologic spectrum of kidney disease in this cohort has not been well described in the recent literature.

Design: Our search of the pathology archives from 2004-2016 yielded 683 kidney biopsies with a diagnosis of IgAN. After review of clinical histories, we identified 25 cases with known liver disease including cirrhosis of any etiology (14 cases), viral hepatitis (5 hepatitis C, 4 hepatitis B), and alcohol abuse ( 2 cases). Pathologic and clinical features were reviewed.

Results: Liver disease was present in 3.7\% of patients with IgAN on kidney biopsy. $56 \%$ of patients were male, and the median age was 50 (range 28-74) years. Most patients presented with nephrotic-range proteinuria (mean proteinuria 4.4 , range $1.6-9.0 \mathrm{gm} /$ day) and renal insufficiency (mean serum Cr 3.6, range $0.9-15.0 \mathrm{mg} / \mathrm{dL}$ ), and many patients had hematuria. By light microscopy, the glomeruli in most cases demonstrated significant mesangial hypercellularity (64\%) and segmental glomerulosclerosis $(76 \%)$. Endocapillary hypercellularity was present in 7 biopsies (28\%), and fibrinoid necrosis +/- 
cellular crescents was present in 3 biopsies $(12 \%)$. These active lesions were identified in cases of hepatitis $\mathrm{C}(\mathrm{HCV})$ infection and alcohol- or HCV-related cirrhosis. Three cases $(12 \%)$ demonstrated a membranoproliferative pattern of injury. By immunofluorescence, mesangial IgA staining was at least $2+$ (of 4 ) intensity in 19 cases (76\%). In addition to mesangial electron dense deposits (EDDs), electron microscopy occasionally detected subendothelial EDDs (5 cases) and subepithelial EDDs (2 cases).

Conclusions: Secondary IgAN in the setting of liver disease is an uncommon but important cause of glomerular disease, and likely underrecognized. Despite the frequent historical description of hepatic IgAN as a mild or "incidental" disease, we found significant glomerular alterations in most cases including significant mesangial hypercellularity, and frequent segmental sclerosis which likely correlated with heavy proteinuria at presentation. Lesions of active GN including endocapillary proliferation, crescents, and necrosis were also present in a significant number of cases with HCV infection and alcohol- or HCV-related cirrhosis.

1623

Myc Expression in 4 Cases of Primary Renal Intravascular Lymphoma

Michelle Don, Ana-Tereza Kabira, Girish Venkataraman, Qin Huang, Christine VanBeek, Cynthia Nast, Mark Haas, Serhan Alkan. Cedars-Sinai Medical Center, Los Angeles, CA.

Background: Primary renal intravascular lymphoma (IVL) is a rare extra-nodal variant of diffuse large B-cell lymphoma (DLBCL). Clinical features and the biology of primary renal IVL have not been well characterized. We studied clinical and immunophenotypic features of 4 cases of primary renal IVL with an emphasis on expression of myc, Bcl2, and Bcl6; as co-expression of these markers implies a worst prognosis. We also evaluated the association of primary renal IVL and EBV by EBER in situ hybridization (ISH). Design: Three cases from our institution and one from an outside institution previously diagnosed as primary renal IVL were retrieved. Clinical features and the complete immunohistochemical (IHC) work-up including results for CD5, CD10, and MUM1were assessed by chart review. Formalin fixed paraffin-embedded blocks of the initial renal biopsy for each case was sent for IHC analysis to identify protein expression of myc, $\mathrm{Bcl} 2$, Bc16. We also evaluated for EBV by EBER-ISH.

\begin{tabular}{|c|c|c|c|c|c|c|}
\hline Case \# & Age/sex & myc & Bcl2 & Bcl6 & CD5 & Type \\
\hline 1 & $57, \mathrm{~F}$ & - & - & + & - & NGC \\
\hline 2 & $69, \mathrm{~F}$ & + & + & - & - & NGC \\
\hline 3 & $70, \mathrm{~F}$ & - & - & - & + & NGC \\
\hline 4 & $56, \mathrm{~F}$ & + & - & + & Unknown & Unknown \\
\hline
\end{tabular}

Results: The average age for each case is 63 (r: 56-70) and all are female. A clinical symptom in all 4 cases includes renal impairment. 3/4 cases have histories of autoimmune disorders (cases 1,2,4). All of the cases show scattered lymphocytic infiltrates in the glomeruli and small vasculature of tubulointerstitial tissues. IHC shows expression of myc in $2 / 4$ cases. Case 2 is positive for myc and $\mathrm{Bcl} 2$, while case 4 is myc and Bcl6 positive. Of the myc negative cases, case 1 is $\mathrm{Bcl} 6$ positive and case 3 is CD5 positive, all other cases are CD5 negative. Cases 1-3 are non-germinal center (NGC) type, negative for CD10 and positive for MUM1 and Bcl6 positive/negative. MUM1 and CD10 were unable to be performed on case 4 . All cases were negative for EBV by EBER-ISH.

Conclusions: Primary renal IVL is a rare subtype of extra-nodal DLBCL. Consistent with the known biology of IVL, all of our primary renal IVL cases showed NGC type. The expression of myc with this entity has not been previously studied. Here we present four cases of primary renal intravascular lymphoma, where two cases have myc protein expression, one with Bcl6 co-expression and one with Bcl2 co-expression, which has been known to imply a worst prognosis. The frequent expression of myc indicates its possible role in the biology of this disease and raises the opportunity for targeted therapy.

\section{Renal Biopsy Data Analysis from Whole Slide Imaging: A Novel} "Holistic" Approach

Beverly E Faulkner-Jones, Charles Law, Milan Rosen, Devin Rosen, Christina I Luffman, Seymour Rosen. BIDMC, Boston, MA; Kitware Inc, Clifton Park, NY.

Background: Pathologists assess and integrate many variables during characterization of a disease process. Using glass slides, these variables are often analyzed sequentially and qualitatively due to the physical and logistic constraints of this traditional medium. Digitization, alignment and analysis of serial whole slide images allow concurrent analysis and integration of multiple parameters with semi-automated quantitation. We have developed a toolkit of methods that can be applied to the renal biopsy.

Design: Serially sectioned renal needle core biopsies were digitized and uploaded onto a digital pathology viewing system. Different anatomic compartments were then quantitatively analyzed. Areas of interest including glomeruli, blood vessels, deposits (oxalate crystals) and cysts (secondary to lithium) were manually segmented. Extracted segmented areas can be used to generate 3-D rendered volumes. Semi-automated quantitative data including cross-sectional area of glomeruli and vessels was extracted from each of the multiple sections in an aligned image stack.

Results: The absolute volumes of tissue structures or deposits can be calculated from rendered 3D-volumes. For example, the relative volume of parenchymal oxalate crystal deposits were calculated as occupying $\sim 7 \%$. The relative volumes of structures may also be calculated using the 3D approach. For example, a 10-fold difference between the volumes of atrophic tubules versus hypertrophic tubules versus tubular cysts was found in lithium nephrotoxicity. We have expanded this approach to the analysis of glomeruli and vessels using two sequential biopsies, "acute" and "healing" phase, from a patient with ANCA-associated glomerulonephritis and concomitant scleroderma vasculopathy. Glomerular area (um2) from multiple contiguous sections plotted as histograms (Fig1a) showed increased variation between glomeruli, but an overall decrease in glomerular size in the second "healing" phase biopsy. The vessels in scleroderma vasculopathy showed marked luminal narrowing that "normalized" with healing (Figlb).

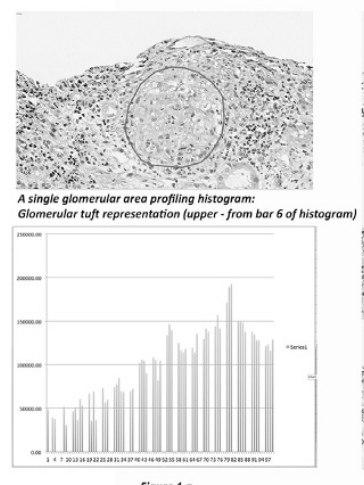

figure 10

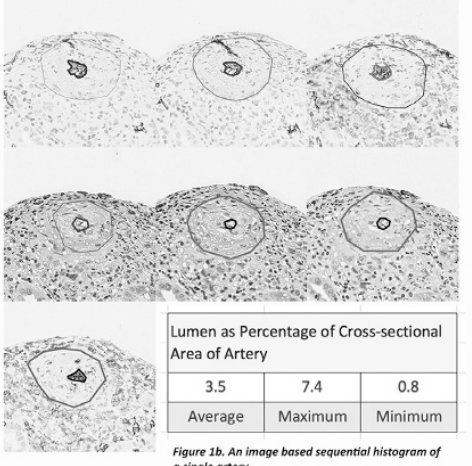

Figure 1b. An im
$a$ single artery.
Conclusions: Digitization and analysis of aligned whole slide images coupled with various quantitative data analytic methodologies can be used to examine renal biopsies in novel ways.

\section{Capsulitis Predicts Poor Kidney Allograft Outcome in T-Cell} Mediated Rejection

Alexander Gallan, W James Chon, Kammi J Henriksen, Anthony Chang. The University of Chicago, Chicago, IL; University of Arkansas, Little Rock, AR.

Background: Acute T-cell mediated rejection (TCMR) is an important cause of renal allograft loss. The Banff classification is based on the degree of lymphocytic infiltration of the tubules (types IA and IB) and arteries (types II and III), which correlates with allograft function and survival. We have observed capsulitis, the presence of lymphocytes between Bowman capsules and parietal epithelial cells, in the setting of TCMR and conducted this study to determine the clinical significance of capsulitis. Design: We identified kidney biopsies with TCMR at our institution from 2010-2015. Exclusion criteria included repeat biopsies from the same patient, presence of $<7$ glomeruli, polyomavirus nephropathy, and Banff type II or III rejection. We assessed for capsulitis, defined as the presence of one or more lymphocytes between Bowman capsules and parietal epithelial cells in a single non-globally sclerosed glomerulus. The 2013 Banff classification for allograft pathology was applied. Clinical metrics were assessed including recovery to baseline creatinine levels after TCMR and progression to dialysis. Data were analyzed using student t-test.

Results: A total of 42 cases of isolated Banff IA or IB TCMR were identified. There was no significant difference in age, sex, graft age, or serum creatinine level at time of biopsy between the cases with and without capsulitis. Patients with capsulitis were more likely to progress to dialysis $(50 \%$ vs $17 \%, \mathrm{p}=0.004)$ and less likely to recover baseline creatinine level $(17 \%$ vs $36 \%, \mathrm{p}=0.039)$. Table 1 summarizes the findings.

\begin{tabular}{|c|c|c|c|}
\hline & $\mathrm{n}$ & Progressed to Dialysis (\%) & Recover Baseline Creatinine (\%) \\
\hline IA with capsulitis & 11 & $5(46 \%)^{*}$ & $1(9 \%)^{* *}$ \\
\hline IA without capsulitis & 14 & $2(14 \%)^{*}$ & $6(43 \%)^{* *}$ \\
\hline IB with capsulitis & 15 & $5(33 \%)$ & $2(13 \%)$ \\
\hline IB without capsulitis & 2 & $1(50 \%)$ & $0(0 \%)$ \\
\hline & & & $\begin{array}{c}{ }^{*} \mathrm{p}=0.045 \\
* * \mathrm{p}=0.033\end{array}$ \\
\hline
\end{tabular}

Conclusions: Capsulitis predicted worse outcomes among those with Banff IA TCMR, as it associated with a higher rate of progression to dialysis and a lower rate of baseline creatinine recovery. Banff IA rejection with capsulitis is clinically as severe as Banff IB rejection.

\section{Light Chain Proximal Tubulopathy of Undetermined} Significance

Toshi Ghosh, Nelson Leung, Samih H Nasr, Mariam P Alexander, Mary E Fidler, Loren P Herrera Hernandez, Lynn D Cornell. Mayo Clinic, Rochester, MN.

Background: Light chain proximal tubulopathy (LCPT) shows accumulation of abnormal crystalline light chain in the proximal tubular cell cytoplasm. Another entity, overflow proteinuria, shows light chain (LC)-restricted protein reabsorption droplets in the tubular epithelial cytoplasm. We describe features of overflow proteinuria with or without tubular injury.

Design: A retrospective analysis was performed from a single institution from 2006 to 2016 of renal biopsies showing single LC-restricted protein reabsorption droplets by immunofluorescence (IF). Biopsies showing intracytoplasmic crystals or atypical casts were excluded.

Results: 14 patients ( 8 M, 6 F) were identified, mean age 58 years (range 40-80). 6 were transplant biopsies. The primary indication for biopsy was increased creatinine $(n=6)$, proteinuria $(n=5)$, and transplant protocol biopsy $(n=3)$. The mean proteinuria was $2.6 \mathrm{~g} /$ day (range 0.11-14.4); the mean creatinine was $1.9 \mathrm{mg} / \mathrm{dL}$ (range 0.5-5.1). Tubular protein reabsorption droplets showed kappa $(n=6)$ or lambda $(n=8)$ by IF. Light microscopy showed acute tubular injury in 4 biopsies, no significant pathology or only arteriosclerosis in 7 , mild interstitial nephritis in 1 , IgA nephropathy in 1 , obesityrelated FSGS in 1. No intracytoplasmic crystals were present by electron microscopy. 
In kappa LC+ biopsies, the median serum kappa free light chain (FLC) was $43 \mathrm{mg}$ / dl (range 1.4-297); in lambda LC+ biopsies, the median lambda FLC was $23 \mathrm{mg} / \mathrm{dl}$ (range 1.5-1540). 10 had a serum monoclonal protein by immunofixation; 11 had urine monoclonal LC. 7 patients (50\%) had plasma cell dyscrasia or lymphoproliferative disorder, including multiple myeloma (MM) $(n=4)$, smoldering $M M(n=1)$, MM in remission $(\mathrm{n}=1)$, and lymphoplasmacytic lymphoma $(\mathrm{n}=1) ; 2$ had amyloidosis. Followup in 12 cases over a median 13.5 months (range 3-88) showed mean creatinine of $1.9 \mathrm{mg} / \mathrm{dL}$ (range $0.8-5.9$ ); 11 cases showed follow-up mean proteinuria of $0.44 \mathrm{~g} /$ day (range 0.06-1.2). Patients were treated for blood cell dyscrasia or amyloidosis, but no patients received treatment for tubular intracytoplasmic LC.

Conclusions: LC-restricted protein reabsorption droplets, with or without acute tubular injury on biopsy, which we term "LCPT of undetermined significance", may present with proteinuria, where excess production of light chains may exceed the reabsorption capacity of tubules, or may be an incidental finding and is commonly associated with serum and urine paraproteins and plasma cell dyscrasias.

1627 Disease Association in Collapsing Glomerulopathy

Francois Gougeon, Harsharan K Singh, J Charles Jennette, Volker Nickeleit. UNCChapel Hill, Chapel Hill, NC.

Background: Collapsing glomerulopathy (CG) is a subtype of FSGS with severe prognostic implications. Most CG is considered to be "idiopathic" with some cases recognized as drug-induced or in the setting of viral infections. Associations with other glomerular diseases have been described, however, comprehensive data on the incidence of such associations is currently lacking. The aim of our study was to characterize renal disease associations in a large cohort of CG patients.

Design: We searched the database of a single nephropathology center for CG in native and transplant kidney biopsies from $01 / 2011$ to $01 / 2016$. Only biopsies with initial diagnosis of $\mathrm{CG}$ were considered. Pathology and submitted clinical data were reviewed. Results: In a total of 8707 biopsies, 352 cases of CG were found (4.0\%). Nephrotic range proteinuria was recorded in 205/250 (82\%) patients. For study purposes cases were classified as "pure" if no other clinically significant renal disease was present. Findings related to disease association in native cases are summarized in table 1.

\begin{tabular}{|l|l|}
\hline CG (total) & $322 / 7527$ \\
\hline Pure & $170(52.8 \%)$ \\
\hline Severe atherosclerosis & $53(16.5 \%)$ \\
\hline MGN & $22(6.8 \%)$ \\
\hline Lupus & $20(6.2 \%)$ \\
\hline DN & $19(5.9 \%)$ \\
\hline TMA & $14(4.3 \%)$ \\
\hline Other & $24(7.5 \%)$ \\
\hline
\end{tabular}

We found a higher rate of $\mathrm{CG}$ in native $(\mathrm{n}=322 / 7527 ; 4.3 \%)$ than in transplant biopsies $(\mathrm{n}=30 / 1180 ; 2.5 \% ; \mathrm{p}=<0.01)$. About half of native cases were classified as "pure" $(\mathrm{n}=170 ; 53 \%)$ whereas remaining patients showed significant renal co-morbidities; mainly severe atherosclerosis $(16.5 \%)$, diabetic nephropathy (DN) (5.9\%), lupus nephritis $(6.2 \%)$, membranous glomerulopathy (MGN) $(6.2 \%)$ and thrombotic microangiopathy (TMA) $(4.3 \%)$. Of note, CG was significantly more common in TMA than in the remaining study cohort $(\mathrm{p}=<0.05)$.

In comparison "pure" CG was only seen in $9 / 30(30 \%)$ of renal allografts. The most frequent co-morbidity was antibody-mediated or mixed rejection with or withou endarteritis in $6(20 \%)$ cases. Other associated co-morbidities included cellular rejection $(10 \%)$, TMA $(10 \%)$ and MGN $(6.7 \%)$. As in natives, CG was also significantly more frequent in TMA cases $(\mathrm{p}=<0.01)$.

Conclusions: We show for the first time in a single center comprehensive study that CG is in greater than $50 \%$ of cases associated with significant renal co-morbidities. Most notably is the concurrence of CG with diseases affecting small vascular blood flow, such as TMAs, severe hypertensive or diabetic arterio-/arteriolosclerosis or acute rejection episodes with capillaritis. Also lupus and MGN show tight associations with CG. This data can serve as basis for further studies into the pathogenesis of CG.

1628 Reducing Inflammation and Oxydative Stress Reverses Established Glomerulosclerosis in Diabetic Kidney Disease

Fabrizio Grosjean, Elena M Yubero-Serrano, Helen Vlassara, Gary E Striker, Fadi Salem. Mount Sinai Medical Center, New York, NY; Fondazione IRCCS Policlinico San Matteo, Pavia, Italy; Reina Sofia University Hospital/University of Cordoba, Cordoba, Spain.

Background: Kidney injury induces progressive glomerulosclerosis (GS) in sclerosisprone mice $(\mathrm{ROP} \mathrm{Os} /+)$. Since their phenotype includes decreased defenses against inflammation (Infl) and oxidative stress (OS), we asked if modulating Infl/OS reduces chronic GS and/or diabetic GS. Pentosan polysulfate (PPS) was used to block responses to extracellular inflammation, pyridoxamine (PYR) to modulate intracellular Infl/OS. Enalapril (EN) was added to all drug regimens. The aim of the study is to determine if reducing Infl/OS in ROP Os/+ mice modifies genetically determined spontaneous GS and/or diabetic GS.

Design: Diabetes, induced with multiple low dose Streptozotocin (STZ) in 8-12 week female ROP Os/+ mice, was treated for 22 weeks; 1) EN $(n=8), 2)$ PYR+EN $(n=8), 3)$ PPS+EN (n=7), and 4) PPS+PYR+EN ( $\mathrm{n}=7)$. Controls included untreated non-diabetic (non-DB) $(\mathrm{n}=7)$ and untreated diabetics $(\mathrm{DB}, \mathrm{n}=8)$. Insulin controlled glucose levels (mean: $250 \mathrm{mg} / \mathrm{dl}$ ).

Results: PPS+PYR+EN treatment reduced diabetic kidney disease (GS and albuminuria). It also reduced chronic kidney disease (CKD) in non-DB. Pro-inflammatory factors were increased in DB; all treatments except EN reduced Monocyte Chemoattractant
Protein-1 (MCP-1) below DB, but tumor necrosis factor (TNF) receptor1 (sTNFR1) was reduced only by PPS+PYR+EN. Anti-Infl/OS defenses were reduced in DB: 1) Advanced glycated end-product receptor 1 (AGER1) was increased to non-DB levels by all except EN; 2$)$ Estrogen Receptor- $\alpha(E R-\alpha)$ was normalized by PYR+EN and PPS+PYR+EN; 3) The nuclear factor erythroid 2-related factor 2 (Nrf2) levels were increased by all treatments vs. DB, and PYR $+E N$ and PYR+PPS+EN normalized Nrf2; and 4) SIRT1 levels were increased above DB and non-DB by PPS+PYR+EN . Conclusions: Controlling inflammation and oxidative stress reverses diabetic kidney disease and $\mathrm{CKD}$ in ROP Os+ mice via modulating the sclerosis-prone phenotype (i.e. albuminuria, GS, increased pro-oxidant/inflammation and decreased anti-oxidant/ inflammation defenses).

1629 The Spectrum of Kidney Diseases Associated with Hepatitis C Virus Infection in the Era of Improved Antiviral Treatment

Shunhua Guo, Agnes Fogo. Vanderbilt University Medical Center, Nashville, TN.

Background: Chronic hepatitis C virus ( $\mathrm{HCV}$ ) infection is a leading cause of chronic liver disease and also frequently causes renal disease. Direct anti-HCV agents have been introduced since 2011. We investigated whether the spectrum of kidney diseases in biopsies of $\mathrm{HCV}$ positive patients has changed since these therapies became available. Design: All kidney biopsies in our laboratory with positive HCV history from January 2008 to January 2016 were identified. The clinical history, laboratory results and renal biopsy diagnoses were reviewed. HCV-related glomerulonephritis (GN) was diagnosed based on morphologic evidence of cryoglobulin, IgM-dominant deposits, clonal shift of kappa/lambda chains and/or characteristic substructure by electron microscopy.

Results: There were $214 \mathrm{HCV}$ positive patients among native kidney biopsies from 2008 to 2016. The HCV positivity rate in kidney biopsies was similar from 2008 to 2011 vs 2012 to 2016 . Among HCV+ patients, the incidence of HCV-related GN was $32 / 78(41.0 \%)$ from 2008 to 2011 and 67/136 (49.3\%) from 2012 to 2016. Diagnostic cryoglobulinemic GN was present in 17/78 (21.8\%) from 2008 to 2011 and 37/136 (27.2\%) from 2012 to 2016 in $\mathrm{HCV}+$ patients. Biopsies with other changes consistent with HCV-related GN constituted 15/78 (19.2\%) from 2008 to 2011 and 30/136 (22.1\%) from 2012 to 2016. Other immune complex GN in $\mathrm{HCV}+$ patients included IgA nephropathy $(1.3 \%$ vs $2.9 \%)$, membranous nephropathy ( $1.3 \%$ vs $2.9 \%)$, postinfectious GN $(5.1 \%$ vs $2.2 \%)$ and fibrillary GN $(2.6 \%$ vs $3.7 \%)$ in $2008-11$ vs $2012-16$. The most common non-immune complex-mediated diseases were diabetic nephropathy $(23.1 \%$ vs $34.6 \%)$ and arterionephrosclerosis ( $15.4 \%$ vs $37.5 \%)$ in $2008-11$ vs $2012-16$. Other occasionally diagnosed diseases in $\mathrm{HCV}+$ patients included lupus nephritis, antiGBM disease, pauci-immune necrotizing crescentic GN, HIV-associated nephropathy, FSGS of NOS, collapsing or tip variants, interstitial nephritis, light chain deposition disease, light chain cast nephropathy and acute tubular injury with calcium phosphate or oxalate crystals.

Conclusions: The spectrum of kidney diseases in biopsied HCV positive patients has not changed in our biopsy practice since the new direct anti-viral agents were introduced. The major pathologic diagnoses are HCV-related GN in nearly half of the patients. Conversely, about half of $\mathrm{HCV}+$ patients have diseases on renal biopsies apparently unrelated to $\mathrm{HCV}$. Whether longer term follow-up of patients receiving novel therapies will shift this spectrum remains undetermined.

\section{Understanding 16 Light Chain Fibrillogenesis/translational Application to Renal Biopsy}

Guillermo A Herrera, Elba A Turbat-Herrera, Jiamin Teng, Bing Liu, Francisco J Rodriguez-Alvarez, Luis del Pozo-Yauner. Louisiana State University Health Sciences Center in Shreveport, Shreveport, LA; National Institute of Genomic Medicine, Mexico, Mexico.

Background: Light chain (AL) derived amyloidosis is the most common form of systemic amyloidosis in the US. AL deposition in the kidneys is most commonly associated with 16 light chains (LC). Immunotyping remains as the most cost-effective option for identifying the amyloid precursor. But, in about $15-20 \%$ of the AL amyloidosis cases, the commercially available antibodies, which target the constant domain $\left(C_{L}\right)$ of LCs, fail to unambiguously identify the AL deposits. Ideally, an antibody for immunotyping $\mathrm{AL}$ amyloid should recognize with high affinity an epitope inherent to the structure of the fibril.

Design: Polyclonal antibodies were generated by immunizing rabbits with five different $\mathrm{KLH}$-conjugated synthetic peptides having the sequence of, respectively, the N-terminal strand (A), b-strand B (B), CDR1 (C), the long loop containing the b strands C and $C^{\prime}(D)$, and part of the FR3 loop (E) of the germline-encoded 16 recombinant variable domain protein 6aJL2. Amyloid fibrils were produced in vitro by incubating the protein at $37^{\circ} \mathrm{C}$ diluted in PBS pH 7.4 with constant agitation. The recognition properties of the antibodies were determined by dot-blots and ultrastructural immunogold labeling, using the 6aJL2 fibrils as target. Tissue samples from patients with renal amyloidosis were also tested using immunogold labeling techniques.

Results: Dot blot and ultrastructural immunogold labeling showed that the five polyclonal antibodies recognized the amyloid fibrils, but not the soluble protein. Antibodies B, D and E showed the strongest recognition of the fibrils in both, dot-blot and immunogold labeling assays.

Conclusions: This study provides a better understanding of specific peptides associated with amyloidogenesis in 16 and demonstrate that in vitro results translate to in vivo improving amyloid detection by enhancing specificity in kidney samples. 
1631 The Role of Transplant Pathologists in Assessing Non-Routine Surgical Specimens from Deceased Donors During Preimplantation Kidney and Liver Biopsies: A 7 Year Retrospective Analysis

Kenneth T Hughes, Shuling Zheng, Stephen C Ward, Fadi Salem. Mount Sinai Medical Center, New York, NY.

Background: Kidney and liver allografts from deceased donors undergo pre-transplant frozen section histology evaluation to determine if the grafts are suitable for donation according to established histological criteria. On-call transplant pathologists also have a pivotal role in diagnosing non-routine, incidental lesions within the donor. Occasionally, malignancy and infection are identified and cause the allograft to be contraindicated. We sought in this retrospective study to analyze these non-routine cases in order to address the role of pathologists in identifying these lesions.

Design: Preimplantation kidney and liver biopsies and non-routine specimens sent to our hospital between December 2009 and August 2016 were evaluated. Non-routine lesions sent during graft assessment were examined and, if present, permanent section diagnoses and consultant opinions were also documented.

Results: We identified 177 non-routine surgical specimens among approximately 3,900 liver and kidney biopsies of deceased donors $(\sim 5 \%)$. Among these, $36(20 \%)$ were renal in origin (primarily benign cysts); $22(12 \%)$ lungs (primarily fibrous or calcified nodules); 21 (12\%) liver (primarily fibrous nodules), 16 (13\%) female genital tract (primarily leiomyomas), 15 (12.5\%) lymph nodes (primarily reactive changes), and 13 (11\%) adrenal glands (primarily adrenocortical adenomas). Overall, $151(85 \%)$ cases were benign, $11(6 \%)$ were suspicious for malignancy, and $6(3 \%)$ were suspicious for infection (3\%). Confirmed on permanent section or by second opinion, malignant diagnoses included 4 renal cell carcinomas, vulvar carcinoma in situ, and lymphoma. A case of histoplasmosis was confirmed with special stains. Challenging benign cases were focal nodular hyperplasia and adrenocortical adenomas.

Conclusions: Transplant pathologists may assess non-routine lesions from potential donors when evaluating preimplantation kidney and liver biopsies. In our study, kidney, lung, liver, female genital tract, lymph node, and adrenal lesions were most often encountered. Occasionally, challenging surgical pathology cases are faced. Hence, it is essential for transplant pathologists to maintain familiarity with select entities in surgical pathology, especially in subspecialized centers, in order to protect recipients from contraindicated grafts or prevent the wasting of valuable donor organs. Additionally, designation of an on-call general surgical pathologist or second reviewer helps ensure an accurate diagnosis.

1632 Complement Activation and ADAMTS13 Suppression May Contribute to the Characteristic Pathological Features in UpshawSchulman Syndrome

Hiroe Itami, Kinta Hatakeyama, Masanori Matsumoto, Shigeo Hara, Makiko Yoshida, Yujiro Asada, Sho Sasaki, Tomoyuki Otani, Tomoko Uchiyama, Tokiko Nakai, Masato Takano, Chiho Ohbayashi. Nara Medical University, Kashihara, Nara, Japan; Kobe University Graduate School of Medicine, Kobe, Hyogo, Japan; Hyogo Prefectural Kobe Children's Hospital, Kobe, Hyogo, Japan; University of Miyazaki, Miyazaki, Japan. Background: Upshaw-Schulman syndrome (USS) is a catastrophic phenotype of congenital thrombotic thrombocytopenic purpura (TTP) characterized by mutations in the gene encoding ADAMTS13. USS is an extremely rare disease, and there have been no systematic studies describing pathological and immunohistological characteristics. Design: We obtained renal biopsies from 5 USS patients with ADAMTS13 mutation, and examined clinicopathological characteristics. To examine the glomerular localization of ADAMTS13 semiquantitatively, double immunohistochemical analysis was performed using antibodies against ADAMTS13, WT1 (podocyte), and CD34 (endothelial cell) in USS cases, 7 acquired TTP cases, and 11 controls. For the assessment of the commitment of complement activation, $\mathrm{C} 4 \mathrm{~d}$ immunoreactivity was also examined.

Results: In USS patient group, average USS age at the time of the renal biopsy was $21.2 \mathrm{yrs}$ (range 9-40) and the mean serum creatinine level was $2.62 \mathrm{mg} / \mathrm{dl}$ (range $0.6-$ 6.16). Except for one case with minor glomerular abnormalities, all cases in USS pathologically demonstrated chronic glomerular changes such as collapsed capillary and duplication of the basement membrane. No thrombus was observed. In two cases, more than half of glomerular segmental sclerosis preferentially localized in perihilar region ( 3 of 3 glomeruli, $100 \% ; 8$ of $12,67 \%$ ). Double immunohistological staining revealed that glomerular ADAMTS13-positive cells were podocytes and endothelial cells. Average number of ADAMTS13-positive cells per 1 glomerulus in USS cases were significantly lower than that in controls $(6.06 \mathrm{vs} 8.55, \mathrm{p}<0.05)$. C4d staining in the glomerular capillary walls were significantly more prevalent in USS than in controls (4 of 5 cases, $80 \%$ vs. 2 of 11 cases, $18 \%$; $<<0.05$ ). There was no significant differences between USS and acquired TTP cases regarding ADAMTS13-positive cells (6.06 vs $5.80, \mathrm{p}=0.81$ ) and $\mathrm{C} 4 \mathrm{~d}$ positivity on glomerular capillary wall ( 4 of 5 cases, $80 \%$ vs. 4 of 7 cases, $57 \%$; $\mathrm{p}=0.58$ ).

Conclusions: From the findings of prominent perihilar distributions of segmental sclerosis, glomerular hilus may be susceptible to the injury in USS. Lower ADAMTS13 expression in podocytes and endothelial cells in USS suggest its possible involvement in the pathogenesis of glomerular lesion. From the immunohistochemical findings of C4d, it is conceivable that complement pathway is activated in USS as in acquired TTP.
1633 Decreasing Incidence of Tubular Basement Membrane Immune Deposits in Polyomavirus Nephropathy

Meghan Kapp, Yasemin Ozluk, Anthony Langone, Harold Helderman, Donald Franklin, Mandeep Grewal, Agnes Fogo. Vanderbilt University Medical Center, Nashville, TN; Instanbul University, Istanbul, Turkey; Nephrology Associates of Chattanooga, Chattanooga, TN.

Background: Polyomavirus nephropathy (PVN) is a major complication of kidney transplantation linked to highly potent immunosuppressive medications, and can cause graft dysfunction and loss. Tubular basement membrane immune deposits (TBMID) involved approximately $50 \%$ of cases in previous series. We assessed the incidence of PVN and impact of TBMID in a more recent cohort.

Design: All PVN renal transplant biopsies in our laboratory over a 15 -year period (2001-2015) were identified. Only cases with adequate biopsy material by CCTT criteria with immunofluorescence microscopy (IF) were included. Electron microscopy was performed in a subset of cases.

Results: $3.4 \%$ of transplant biopsies (156/4569) from 122 patients were diagnosed as PVN. IF was assessed in 129 of 156 tissues (83\%), with TBMID detected in 27 ( $21 \%$ ) of these cases. Among these 27 TBMID positive cases, the extent of staining of tubules by IF was on average $23 \%$ (range $5-75 \%$ ). C3 positivity of TBM was present in $76 \%$, followed by $\operatorname{IgG}(47 \%), \operatorname{IgM}(39 \%), \operatorname{IgA}(13 \%)$, and $\mathrm{C} 1 \mathrm{q}(11 \%)$ in various combinations. EM specimens available in 65 cases $(42 \%)$ did not reveal deposits in glomeruli or compartments other than TBM.

There were no differences in demographic data, type of transplantation, primary kidney disease, the average time after transplantation to the first biopsy, presence of acute rejection, or initial serum creatinine levels in PVN cases with or without TBMID. In some patients with repeated biopsies, PVN-associated TBMID resolved, despite persistent SV40 positivity

Conclusions: Former studies demonstrated TBMID in about half of PVN cases, thus suggesting the presence of TBMID is common and a helpful feature in diagnosis. However, in our series, TBMID were present much less frequently (21\%). The lower incidence of TBMID in our patients may reflect earlier detection with less severe PVN. This also suggests the possibility that viral antigens could play an important role in the formation of in situ antigen-antibody complexes. Whether TBMID positivity in PVN has prognostic implications awaits further study.

1634 Cisplatin-Induced Nephrotoxicity and HIV Associated Nephropathy : Mimickers of Myeloma Like Cast Nephropathy

Muhammad S Khurram, Warda Ibrar, Ahmed Alrajjal, Hong Qu. St John Hospital and Medical Center, Detroit, MI.

Background: True myeloma cast nephropathy is an obstructing disorder of renal tubules, caused by precipitation of Bence Jones proteins with TammHorsfall protein and other filtered proteins in the form of angulated and brittle casts.Myeloma-Like Cast Nephropathy (MLCN) have been reported in the literature to occur in various primary renal and non-renal diseases including some neoplastic processes.

Design: We present series of three rare cases of cast nephropathy in HIV patients without plasma cell dyscrasia. A 30 -year-old male admitted to the hospital with facial cellulitis, skin biopsy revealed coccidiodomycosis and second patient is a 31 -year-old male who presented with shortness of breath, productive cough, and recent 50 pound weight loss chest radiograph and CT scan showed bilateral ground $\neg$ glass opacities consistent with pneumocystis jiroveci pneumonia. Third patient patient was treated with cisplatin-based chemotherapy secondary to squamous cell carcinoma.

Results: Light Microscopy: First two cases revealed foci of tubular cystic dilatation and diffuse tubular dilatation containing hyaline casts, with intense neutrophilic cellular reaction. Focal interstitial inflammatory infiltrate including lymphocytes, plasma cells, neutrophils was seen. The third case showed acute tubular injury and cast formation The casts elicited a cellular reaction of macrophages and neutrophils.

Electron Microscopy:First two cases showed globally sclerotic glomeruli with ultrastructural findings showing podocytes with extensive foot process fusion. The mesangium showed expansion by increased matrix and mesangial cell processes. Glomerular basement membrane was slightly thickened. Third case showed irregular casts appearing dark blue in toluidine blue, composed of amorphous or granular material of low density admixed with scattered high electron-dense globules and membrane debris.

Immunofluorescence Microscopy:Direct immunofluorescence stains were performed using a panel of ten anti sera. It failed to demonstrate monoclonal restriction. All glomeruli were negative for albumin, fibrinogen, C1q, C3, C4.IgA, IgM, Kappa and Lambda light chain stains.

Conclusions: Myeloma $\neg$ like cast nephropathy and true myeloma cast nephropathy pose similar destructive effects on renal parenchyma. This new pattern of HIV $\neg$ related nephropathy should be recognized and in the absence of monoclonal gammopathy, HIV status of the patients should be checked.

1635 D-Penicillamine Partially Amiliorates Tubulointerstitial Fibrosis by Inhibition of Lysyl Oxidase-Like 2

Jisup Kim, Sung-Eun Choi, Nara Jeon, Hyeon Joo Jeong, Beom Jin Lim. Yonsei University College of Medicine, Seoul, Korea.

Background: Tubulointerstitial fibrosis is a common end point of chronic kidney diseases. Lysyl oxidase-like 2 (LOXL2) is a molecule known to be related with invasive growth and metastasis of malignant neoplasms. Recently, it is reported that LOXL2 is also responsible for organ fibrosis. Little is known about the expression of LOXL2 in kidney and its contribution to the tubulointerstitial fibrosis. In this study, we evaluated the expression of LOXL2 in human and mouse kidney and the role of LOXL2 in the progression of renal tubulointerstitlal fibrosis. 
Design: The expression of LOXL2 was evaluated in paraffin-embedded human and mouse kidney by immunohistochemistry. Then, double immunofluorescence microscopy of LOXL2 and podocyte marker or proximal tubular marker was performed. LOXL2 was also evaluated in cultured human proximal tubular cells (HK-2 cells), canine distal tubular cells (MDCK cells) and conditionally immortalized human podocytes. Tubulointerstitial fibrosis was induced in CD1 mice by intraperitoneal injection of folic acid $(240 \mathrm{mg} / \mathrm{kg})$. The mice were sacrificed 4 to 6 weeks after folic acid injection, and renal expression of LOXL2 was evaluated. To evaluate the effect of LOLX2 inhibition, folic acid-injected mice were treated with d-penicillamine $(150 \mathrm{mg} / \mathrm{kg})$ or vehicle twice a week. Four weeks after foclid acid injection, the mice were sacrificed and the kidneys were evaluated for the degree of tubulointerstitial fibrosis.

Results: LOXL2 expression was observed in tubular epithelial cells and glomeruli along the glomerular capillary loops in both human and mouse kidney. Most of the tubular expression were localized to the proximal tubules. In the glomerlui, LOXL2 was localized to podocytes. By western blot, LOXL2 was deteted in HK-2 cells and human podocytes, but not in MDCK cells supporting the above results. After inducing tubulointerstitial fibrosis in CD1 mice, LOXL2 mRNA significantly increased compared to vehicle-injected mice. Some mice treated with d-penicillamine showed preserved renal volume, less amount of tubulointerstitial fibrosis and decreased expression of TGF- $\beta$ and related molecules.

Conclusions: LOXL2 expression was observed in the proximal tubular epithelial cells and podocytes of human and mouse kidney, and related with the progression of tubulointerstitial fibrosis. D-penicillamine, an inhibiotr of LOLX2, demonstrated anti-fibrotic effect. Further evaluation on LOXL2 in kidney and its contribution to the tubulointerstitial fibrosis is needed.

\section{Thrombotic Microangiopathy in the Kidney, Pathologic Indicators of Disease Severity}

Satoru Kudose, Joseph Gaut. Washington University in St. Louis, St. Louis, MO

Background: Microvascular endothelial injury and thrombosis define thrombotic microangiopathy (TMA), a pathologic diagnosis associated with numerous etiologies. Clinically, TMA presents with microangiopathic hemolytic anemia (MAHA), thrombocytopenia, and acute kidney injury. There is no consensus regarding TMA classification in modern clinical practice. This study investigates the relationship between TMA pathology, clinical presentation, outcome and etiology.

Design: Retrospective review of 45 native renal biopsies with a pathologic diagnosis of TMA without immune-complex mediated disease was performed. Each biopsy was semi-quantitatively assessed for morphologic changes of TMA blinded to clinical data. Clinical findings were determined from the electronic medical record.

Results: Patients ranged from 4 to 73 years old (mean 43.87 years) and $16(35.6 \%)$ were male. TMA etiology included hypertension (24.4\%), atypical hemolytic uremic syndrome (aHUS) (20\%), medications $(11 \%)$, bone marrow transplant $(11 \%)$, scleroderma $(8.9 \%)$, and unknown $(8.9 \%)$. Arteriolar mucoid intimal edema ( $0.792 \mathrm{vs.}$ $0.381, \mathrm{OR}=5.90, \mathrm{p}=0.000694)$, arteriolar fibrinoid change $(0.625$ vs. $0.286, \mathrm{OR}=4.02$, $\mathrm{p}=0.0362)$, and onion skinning $(0.0476$ vs. $0.417, \mathrm{OR}=13.5, \mathrm{p}=0.00517)$ were more frequent in patients with dialysis-requiring renal failure. Of biopsies with arteriolar TMA, $90 \%$ showed glomerular mesangiolysis, endotheliosis, or thrombi. Ultrastructural subendothelial edema $(0.522$ vs. $0.857, \mathrm{p}=0.0441)$ and arteriolar fibrinoid change ( 0.250 vs. $0.586, p=0.0596)$ were more frequent in cases with MAHA. MAHA was present in $64.4 \%$ of cases, and those with MAHA were more likely to undergo dialysis $(0.688$ vs. 0.345 , Odds ratio $(O R)=4.04, p=0.0345)$. There was no correlation with pathology and TMA etiology.

Conclusions: Vascular pathology is independently predictive of need for dialysis (multivariate logistic regression, $\mathrm{p}<0.05$ ), although mucoid intimal edema and fibrinoid change were not independent of each other ( $r=0.673$, Spearman's). Vascular lesions commonly occurred with glomerular pathology, arguing for a continual disease spectrum as opposed to two distinct pathologic groups. The vascular pathologic lesions were strong predictors of dialysis independent of and more powerful than MAHA and their presence suggests more severe disease. Prospective evaluation of these results in a larger cohort is needed to assess their prognostic utility.

\section{De Novo Immune Complex Deposition in Kidney Allografts}

Isaac E Lloyd, Faris Ahmed, Monica P Revelo, Mazdak Khalighi. University of Utah, Salt Lake City, UT.

Background: Glomerulonephritis in kidney allografts can include both recurrent and de novo processes. Reported de novo immune complex(IC)-mediated diseases include membranous glomerulopathy (MG), IgA nephropathy (IgAN) and infection-related glomerulonephritis (IRGN). We performed the following study to better characterize de novo IC deposition in kidney allografts.

Design: 30 patients with glomerular deposits not attributable to the native disease were identified from 2009-2015 (biopsy incidence 7\%). Pathologic and clinical features were reviewed.

Results: The most common pattern of glomerular injury was mesangial proliferative GN (mesGN) in $70 \%$ of biopsies, followed by membranoproliferative GN (MPGN, $23 \%) .2$ patients (7\%) had MG.

A concurrent or recent infection was identified in $9(30 \%)$ patients, including hepatitis $C$ virus infection in 1.21 patients had no clinical features of infection; however, 2 showed mesGN with $\mathrm{C} 3$ deposits favoring resolving IRGN. In those without IRGN, 6 had IgAN, 2 had MG and 1 had mesGN with IgG-dominant deposits and a long history of Crohn disease. ANA was positive in 1 patient, who also had a history of PTLD. Biopsy showed MPGN with IgM-dominant deposits with organized substructure. Cryoglobulin testing was not performed. Serologic studies were negative in the remaining patients who were tested.
$10(33 \%)$ patients showed glomerular IC deposition that could not be explained clinically and did not fit any specific form of GN. 7 showed mesGN, while 3 demonstrated an MPGN pattern that were either IgG dominant (8) or IgM dominant (2). These patients were diagnosed a median of 4.25 years after transplant (range: 0.3 13 years) and $5(50 \%)$ had concurrent antibody-mediated rejection (AMR). 5 patients were tested for donor specific antibodies, which were positive in $4(80 \%)$. In contrast, IC deposition was diagnosed a median of 8.7 years post-transplant for the remaining patients and only $3(15 \%)$ had concurrent AMR. DSA results were available in 12 patients and were positive in $2(17 \%)$.

Conclusions: De novo IC deposition is uncommon, accounting for $7 \%$ of transplant biopsies reviewed at our institution. Most patients had a clearly defined disease process such as IgAN, IRGN or MG; however, one third of patients had IC deposition that is difficult to classify. Possible explanations include subclinical infection or a form of chronic humoral injury. Further studies are required to better characterize the origin of deposits in such cases.

$1638 \quad$ Non-Neoplastic Kidney Chronic Injury Score Predicts PostOperative Renal Function in Radical Nephrectomy Specimens

Miao Lu, Deepak K Pruthi, Corey Knickle, Thomas B McGregor, Ian W Gibson. University of Manitoba, Winnipeg, MB, Canada; University of Texas Health Sciences Centre, San Antonio, TX.

Background: The non-neoplastic kidney change may provide important information to identify patients at risk to develop significant decline in post-operative renal function. Design: We retrospectively reviewed all patients undergoing RN for RCC measuring $\leq 10 \mathrm{~cm}$ with a functioning contralateral kidney between January 1, 2011 - May 1,2015. The non-neoplastic kidney histopathologic changes were graded, based on the Banff 97 criteria for chronic allograft injury, for chronic glomerular $(\mathrm{G})$, tubulointerstitial (IFTA), arterial (Art), and arteriolar changes (Arl). Univariate analyses were conducted with Spearman correlation coefficients and ANOVA. Factors significantly associated with post-operative eGFR were entered into multivariable regression (MVR) models. Separate regression models were created for the clinical and histological factors. Predictive performance was evaluated with the r-square statistic. Estimated glomerular rate (eGFR) was calculated using chronic kidney disease epidemiology collaboration formula for up to two year values.

Results: In total 167 patients met the inclusion criteria. Mean age, Charlson comorbidity score, and tumor size were $61,2.6$, and $6.2 \mathrm{~cm}$, respectively. The group consisted of diabetics $(26 \%)$, hypertensives $(60 \%)$, and smokers $(35 \%)$. Mean pre-op eGFR and 24-month eGFR were 78 and $51 \mathrm{~mL} / \mathrm{min} / 1.73 \mathrm{~m}^{2}$, respectively. Severe NNK changes were found in $11 \%$ of patients with normal pre-operative eGFR. New kidney disease was common (54\%); five patients progressed to dialysis and nine died during followup. NNK changes were significantly associated post-op eGFR at 1 year $\left(\mathrm{R}^{2}=0.52\right)$ and 2 years $\left(\mathrm{R}^{2}=0.5\right)$. Severe $\mathrm{G}$ and IFTA were associated with an independent eGFR change of decline of $-28(\mathrm{p}<0.001)$ and $-16 \mathrm{~mL} / \mathrm{min} / 1.73 \mathrm{~m}^{2}(\mathrm{p}=0.0005)$. Art $(\mathrm{p}=0.08)$ and Arl $(\mathrm{p}=0.387)$ changes were not associated with eGFR changes. Age $(\mathrm{p}<0.0001)$, body mass index $(\mathrm{p}=0.0039)$, and age-adjusted Charlson co-morbidity $(\mathrm{p}=0.0434)$ were significantly associated with post-op eGFR. Using MVR modeling, a calculator was developed which predicted post-op eGFR.

Conclusions: Using age, glomerular, and tubulointerstitial histopathologic variables alone, the post-operative eGFR can be accurately predicted. Grading the chronic injury of the non-neoplastic kidney in nephrectomy specimens provides accurate prediction of post-operative renal function and may guide therapeutic intervention.

1640 A Demographic and Pathologic Analysis of 100 Consecutive Renal Core Biopsies in a Tertiary Laboratory in Johannesburg

Washington Mudini, Pulane N Mosiane. University of Witwatersrand, Johannesburg, Gauteng, South Africa; National Health Laboratory Service, Johannesburg, Gauteng, South Africa.

Background: Renal disease remains an important cause of morbidity and mortality in South Africa and very little is known about the patterns of disease in the region. A national renal data registry is yet to be established and studies on biopsy-proven renal disease are scarce. In this study, we assessed the demographics and disease patterns of patients who underwent a renal biopsy with the aim of describing the patterns of disease. Design: A retrospective review of 100 consecutive renal biopsy reports from the National Health Laboratory Service (NHLS) Charlotte Maxeke Hospital in Johannesburg from January to June 2013 was undertaken. The renal biopsies were studied by light, immunofluorescence and electron microscopy. The data was collected and stratified according to age, gender, clinical presentation and histological diagnosis.

Results: Of the 100 biopsy reports reviewed, 31 were from transplant patients and were excluded from analysis. Of the remaining 69 native renal biopsies, 34 were from males and 35 from females ( 65 blacks and 4 whites). The mean age at biopsy was 28.7 years (range 3 months to 63 years). $78.2 \%$ of patients were under 40 years of age and $29 \%(20 / 69)$ were HIV positive. The commonest clinical indication for biopsy was nephrotic syndrome which accounted for $36.2 \%(25 / 69)$ of cases followed by renal dysfunction $17 / 69(24.6 \%)$ and systemic lupus erythematosus $17.4 \%(12 / 69)$. Minimal change disease accounted for the most cases of primary glomerulonephropathies with $17.4 \%(12 / 69)$ cases, followed by focal segmental glomerular sclerosis $13.8 \%(9 / 69)$. Lupus nephritis was the most frequent secondary glomerular disease 14/69 (20.3\%) and was also the most frequent cause of the nephrotic range proteinuria $17.2 \%(5 / 29)$. Membranous glomerulopathy and HIV-associated nephropathy each accounted for $4 \%(3 / 69)$ of cases.

Conclusions: The most common reason for biopsy in our population was nephrotic syndrome. A fifth of the patients in our study population were HIV positive and this confirms our suspicion that HIV is a major cause of kidney disease. This report represents 
an epidemiological overview on biopsy proven renal disease from one specific tertiary laboratory in a Sub Saharan country. This will aid in the development of not only a national register, but also a register for the region.

\section{Post-Transplant Thrombotic Microangiopathy Associated with} a Monoclonal Gammopathy: A Case Series

Aishwarya Ravindran, Ronald S Go, Fernando C Fervenza, Sanjeev Sethi. Mayo Clinic, Rochester, MN

Background: Thrombotic microangiopathies (TMA) are rare, potentially lifethreatening conditions caused by small-vessel-platelet microthrombi. Monoclonal gammopathy may act as a potential trigger in the pathogenesis of TMA. We performed a retrospective study to determine the prevalence of monoclonal gammopathy in posttransplant patients with TMA.

Design: We included adults (age $\geq 18$ years) from 2000-2016 with a clinical diagnosis of TMA who met all of the following criteria: i) microangiopathic hemolytic anemia and thrombocytopenia or histologic evidence of TMA in post-transplant patients, ii) absence of a coagulopathy and a negative direct antiglobulin test, iii) screened for monoclonal gammopathy, and iv) negative for antibody-mediated rejection including negative donor-specific antibodies.

Results: 19 patients met the study criteria. A monoclonal protein was detected in 5 patients $(26.3 \%)$. Of these 5, $2(40 \%)$ received autologous stem cell transplant for multiple myeloma (MM) and non-Hodgkin lymphoma, $2(40 \%)$ allogeneic stem cell transplant for chronic lymphocytic leukemia and MM , and $1(20 \%)$ kidney transplant for end-stage renal disease, respectively. The median age at diagnosis of TMA in patients with monoclonal protein was 63 years (range: 42-67), and the majority were males $(3,60 \%)$. Among patients $\geq 50$ years, the prevalence of monoclonal gammopathy was $27.3 \%(\mathrm{n}=11)$, which is approximately 8 -fold higher than the expected rate in this population (4.2\%). Among the 5 patients with monoclonal gammopathy, $3(60 \%)$ were drug induced (Melphalan, Tacrolimus, Sirolimus, respectively), 1 (20\%) infection and $1(20 \%)$ could not be classified. The median serum creatinine at the time of TMA diagnosis was $3.4 \mathrm{mg} / \mathrm{dL}$ (range: $2.9-5.3$ ). Renal biopsy was performed in 3 cases and showed glomerular microthrombi, mesangiolysis, and double contours. $1(20 \%)$ received PLEX, $2(40 \%)$ received PLEX and steroid/immunosuppressants, and $2(40 \%)$ received steroid/immunosuppressants. 4 patients $(80 \%)$ required dialysis during the course of the disease, of which 2 progressed to end-stage renal disease, 1 had renal allograft dysfunction, and 1 had improved renal function at final follow-up.

Conclusions: Our study shows an unexpectedly high prevalence of monoclonal gammopathy in post-transplant patients with TMA suggesting a potential association. However, more studies are required to determine the underlying mechanisms of TMA in the presence of a monoclonal protein in transplant recipients.

1642 Post-Transplant Thrombotic Microangiopathies: A Case Series Aishwarya Ravindran, Ronald S Go, Fernando C Fervenza, Sanjeev Sethi. Mayo Clinic, Rochester, MN.

Background: Thrombotic microangiopathies (TMA) are a heterogenous set of conditions that result in microvascular thrombosis. We performed a retrospective, single-institution study to determine the causes and characteristics of TMA in posttransplant patients.

Design: We included adults (age $\geq 18$ years) from 2000-2016 with a clinical diagnosis of TMA who met all of the following criteria: i) microangiopathic hemolytic anemia and thrombocytopenia or histologic evidence of TMA in post-transplant patients, ii) absence of a coagulopathy and a negative direct antiglobulin test, and iii) negative for antibody-mediated rejection including negative donor-specific antibodies.

Results: 22 patients met the study criteria. The classification of TMA and associated causes of transplant are described in the table.

\begin{tabular}{|l|l|}
\hline Classification of TMA; N=22 & Type of Transplant \\
\hline $\begin{array}{l}\text { atypical Hemolytic Uremic } \\
\text { Syndrome; } \mathrm{n}=3\end{array}$ & $\begin{array}{l}\text { i) Auto PBSCT: 1, (NHL)ii) Allo PBSCT: 1, (AML)iii) Kidney: } \\
\text { 1, (obstructive uropathy) }\end{array}$ \\
\hline $\begin{array}{l}\text { TMA (Drug); n=4[2 } \\
\text { Tacrolimus, 1 Sirolimus, 1 } \\
\text { Melphalan] }\end{array}$ & $\begin{array}{l}\text { i) Auto PBSCT: 1, (MM)ii) Allo PBSCT: 2, (AML, MM)iii) } \\
\text { Kidney: 1, (cyclosporine toxicity) }\end{array}$ \\
\hline TMA (Infection); n=1 & i) Allo PBSCT: 1, (CLL) \\
\hline TMA (Unclassified); n=14 & $\begin{array}{l}\text { i) Auto PBSCT: 2, (NHL)ii) Allo PBSCT: 10, (AML:3, ALL:4, } \\
\text { CLL:1, HL:1, NHL:1)iii) Kidney: 2, (glomerulonephritis, } \\
\text { unknown etiology) }\end{array}$ \\
\hline $\begin{array}{l}\text { Abbreviations: PBSCT: peripheral blood stem cell transplant, AML: acute myeloid leukemia, } \\
\text { ALL: acute lymphoblastic leukemia, CLL: chronic lymphocytic leukemia, HL: Hodgkin } \\
\text { lymphoma, MM: multiple myeloma, NHL: non-Hodgkin lymphoma }\end{array}$ \\
\hline
\end{tabular}

The median age at diagnosis was 51.5 years (range: 19-76), and the majority were males $(14,63.6 \%)$. The median serum creatinine at the onset of TMA was $2 \mathrm{mg} / \mathrm{dL}$ (range: 0.8-8.6). Renal biopsy was performed in 7 cases and showed glomerular microthrombi, mesangiolysis, and double contours. The median follow-up was 29.6 months (Range: $0.2-458.2$ ). At final follow-up, the median serum creatinine was $1.4 \mathrm{mg} / \mathrm{dL}$ (Range: $0.8-3)$. Of the 22 patients: $1(4.5 \%)$ received PLEX, $12(54.5 \%)$ received PLEX and steroid/ immunosuppressants, and $9(41.0 \%)$ received steroid/immunosuppressants. Of the 22 patients, 7 patients $(31.8 \%)$ required dialysis during the course of TMA, of which 3 progressed to ESRD, 2 continued to have renal allograft dysfunction, and 2 had improved renal function at final follow-up.

Conclusions: Our study describes the etiology and clinical course of TMA in posttransplant patients. TMA is an uncommon but important cause of renal dysfunction in post-transplant patients.
1643 C3GN with a Severe Crescentic Phenotype

Aishwarya Ravindran, Fernando C Fervenza, Mariam P Alexander, Sanjeev Sethi. Mayo Clinic, Rochester, MN.

Background: C3 glomerulopathy is characterized by glomerular accumulation of C3 with minimal or no Ig. Based on the EM, it is classified into $\mathrm{C} 3$ glomerulonephritis (C3GN) or dense deposit disease (DDD). C3GN most commonly presents with an MPGN pattern of injury. In a small subset of patients, C3GN may present with a severe crescentic GN. There are no series describing the clinicopathologic findings of crescentic $\mathrm{C} 3 \mathrm{GN}$

Design: We identified 79 patients from 2010-2016 with a diagnosis of C3GN. Patients whose kidney biopsies showed cellular crescents involving $\geq 50 \%$ of the glomeruli were included.

Results: $4(5.1 \%)$ patients met the study criteria. The median age at diagnosis was 20.5 years (range: $12-52)$, the majority were females $(75 \%)$. At onset, the median serum creatinine was $2.4 \mathrm{mg} / \mathrm{dL}$ (range: $1.3-6.2$ ), the median eGFR was $36 \mathrm{ml} / \mathrm{min} / 1.73 \mathrm{~m}^{2}$ (range: 7-51), and the median urinary protein was $4068 \mathrm{mg} / 24$ hours (range: 19896147), respectively. All patients had hematuria, low C3, and normal C4. Evaluation of the complement pathway revealed a mutation in Factor $\mathrm{H}$ (1 patient), a mutation/ polymorphism in $\mathrm{MCP} / \mathrm{CD} 46$ (1 patient), elevated CBb levels (1 patient), and tests were not done in 1 patient (deceased). On renal biopsy, the main finding was that of severe crescentic and necrotizing GN. The median percentage of cellular crescents was $54 \%$ (range: 50-82\%). IF studies showed bright glomerular C3 (3+) staining with no or scant staining for Ig. EM studies showed mesangial and capillary wall deposits, including subepithelial deposits in 2 cases. 3 cases were treated with steroids/immunosuppressants, 1 patient died 5 months after biopsy. The median follow-up was 11.7 months (range: $0.2-65.6$ ). At final follow-up, the median serum creatinine was $1.9 \mathrm{mg} / \mathrm{dL}$ (range: 1.3 2.6), the median eGFR was $47 \mathrm{ml} / \mathrm{min} / 1.73 \mathrm{~m}^{2}$ (range: $24-50$ ), and the median urine protein was $2455 \mathrm{mg} / 24 \mathrm{hrs}$ (range: $750-2836$ ), respectively. We compared the C3GN patients with 2 DDD patients with similar crescentic subtype. Patients with DDD $(n=2)$ had $100 \%$ crescents on renal biopsy and at onset, the median serum creatinine was $3.6 \mathrm{mg} / \mathrm{dL}$ (range: $3.5-3.7$ ), the median eGFR was $18 \mathrm{ml} / \mathrm{min} / 1.73 \mathrm{~m}^{2}$ (range: $15-20$ ), respectively. Both these patients progressed to ESRD and received kidney transplant after a median duration of 22.3 months (range: 20.3-24.3) from the time of kidney biopsy. Conclusions: A subset of $\mathrm{C} 3 \mathrm{GN}$ present with rapidly progressive loss of kidney function and are characterized by severe crescentic GN. It is important to recognize this subset of $\mathrm{C} 3 \mathrm{GN}$ and differentiate it from other causes of crescentic GN including anti-GBM and ANCA-GN. Further studies are required to determine the pathophysiology of crescentic $\mathrm{C} 3 \mathrm{GN}$.

\section{BK Virus Disease Severity and Predictors of Graft Outcomes in Renal Transplants: A Single Institution Experience}

Melissa Swee, Sook-kyung Kwon, M L Sanders, Mony Fraer, Sarat Kuppachi, Prerna Rastogi. University of Iowa, Iowa City, IA.

Background: BK virus nephropathy (BKN) is an important cause of graft failure after renal transplant. We sought to identify morphologic predictors corresponding to disease severity that would help direct early and appropriate therapeutic interventions. Design: 20 cases with biopsy proven BKN were identified over 12 years from 1031 transplants. Using histologic criteria that have been described previously biopsies were re-evaluated and classified into early, florid and late fibrotic stages. IHC stain for Polyomavirus large T-antigen (SV-40) was also evaluated.

Results: Biopsy stage did not significantly correlate with graft failure $(\mathrm{p}=0.49)$, viral load $(\mathrm{p}=0.38)$ or BK viral resolution $(\mathrm{p}=0.16)$. Key patient data are in TABLE1.

\begin{tabular}{|c|c|c|c|c|c|c|}
\hline $\begin{array}{l}\text { Age/ } \\
\text { Sex }\end{array}$ & Stage & \begin{tabular}{|l} 
S.Cr.at \\
biopsy \\
(mg/dL) \\
\end{tabular} & $\begin{array}{l}\text { BKviral load } \\
\text { (copies/mL) }\end{array}$ & $\begin{array}{l}\text { Viremia } \\
\text { resolved }\end{array}$ & $\begin{array}{l}\text { Graft } \\
\text { Failure }\end{array}$ & $\begin{array}{l}\text { S.Cr. at BK } \\
\text { resolution }\end{array}$ \\
\hline $66 / \mathrm{M}$ & EARLY & 2.4 & 34500 & $\mathrm{~N}$ & $\mathrm{~N}$ & 2.3 \\
\hline $57 / \mathrm{M}$ & FLORID & 4.3 & 80900 & $\mathrm{~N}$ & $\mathrm{Y}$ & 10 \\
\hline $19 / \mathrm{M}$ & EARLY & 1.47 & 1900 & $\mathrm{~N}$ & $\mathrm{~N}$ & 1.9 \\
\hline $57 / \mathrm{M}$ & FLORID & 3.8 & 9757615 & $\mathrm{Y}$ & $\mathrm{N}$ & 2.52 \\
\hline $59 / \mathrm{F}$ & FLORID & 1.8 & 92200 & $\mathrm{~N}$ & $\mathrm{~N}$ & 6.3 \\
\hline $7 / \mathrm{M}$ & ADVANCED & 4.2 & 38500 & $\mathrm{Y}$ & $\mathrm{Y}$ & 10 \\
\hline $47 / \mathrm{M}$ & EARLY & 3.8 & 29400 & Y & $\mathrm{N}$ & 4.15 \\
\hline $68 / \mathrm{M}$ & FLORID & 1.5 & 88200 & $\mathrm{Y}$ & $\mathrm{N}$ & 2.55 \\
\hline $56 / \mathrm{M}$ & ADVANCED & 4.2 & 16700 & $\mathrm{Y}$ & $\mathrm{N}$ & 1.3 \\
\hline $40 / \mathrm{M}$ & FLORID & 2.3 & 71500 & $\mathrm{Y}$ & $\mathrm{N}$ & 2 \\
\hline $17 / \mathrm{M}$ & FLORID & 1.3 & 19300 & $\mathrm{Y}$ & $\mathrm{N}$ & 0.96 \\
\hline $65 / \mathrm{M}$ & FLORID & 1.5 & 2753000 & $\mathrm{Y}$ & $\mathrm{N}$ & 1.5 \\
\hline $55 / \mathrm{M}$ & ADVANCED & 4.3 & $>5000000$ & $\mathrm{Y}$ & $\mathrm{Y}$ & 10 \\
\hline $36 / \mathrm{F}$ & EARLY & 1.9 & 7795 & $\mathrm{Y}$ & $\mathrm{Y}$ & 10 \\
\hline $63 / \mathrm{F}$ & EARLY & 1.6 & $<5000$ & $\mathrm{Y}$ & $\mathrm{N}$ & 1.5 \\
\hline $63 / \mathrm{M}$ & FLORID & 2.9 & 43100 & $\mathrm{Y}$ & $\mathrm{Y}$ & 10 \\
\hline $69 / \mathrm{M}$ & FLORID & 3.2 & 12200 & $\mathrm{Y}$ & $\mathrm{N}$ & 2.1 \\
\hline $53 / \mathrm{F}$ & ADVANCED & 2.2 & 200000 & $\mathrm{Y}$ & $\mathrm{N}$ & 1.6 \\
\hline $40 / \mathrm{M}$ & FLORID & 7.5 & 9200 & $\mathrm{Y}$ & $\mathrm{N}$ & 4.7 \\
\hline $32 / \mathrm{M}$ & ADVANCED & 2.3 & 4701024 & $\mathrm{Y}$ & $\mathrm{N}$ & 3 \\
\hline
\end{tabular}

Representative biopsy findings are in FIGURE1. 


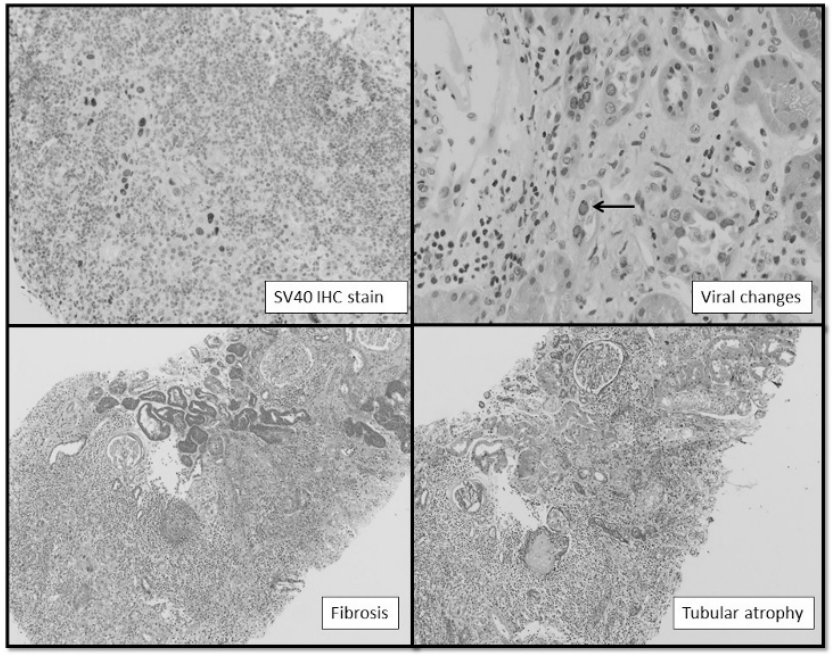

Conclusions: Our study suggests that severity of BKN does not correlate with BK viral load and the degree of histopathologic changes seen in BKN do not definitively predict graft outcomes. Nevertheless, it is important to identify early markers for BK viral infection in transplant patients to prevent unfavorable graft outcome.

1647 Low Ratio of CD68/Kidney Injury Molecule-1 (KIM-1) Expression in the Proximal Tubules of Renal Biopsies Is Associated with Worse Renal Recovery at 6 Month Follow-Up

Nicholas D Ward, Ping L Zhang. Beaumont Health, Royal Oak, MI.

Background: Acute tubular injury (ATI) is often the cause of renal dysfunction, but currently there is no good predictive index for renal functional recovery. Based on our previous studies, KIM-1 has biphasic biology during ATI - KIM-1 works as phagocytic factor involved in proximal tubular repair during mild to moderate ATI but in severe/ prolonged ATI, KIM-1 may shift to promote fibrosis. CD68 is another phagocytic factor - upregulated in early injury, but we find that it disappears when ATI becomes severe. We examined the relative expression of CD68 and KIM- 1 as reflected by staining intensity in renal biopsies and correlated the CD68/KIM-1 ratio with renal function recovery one month and 6 months after renal biopsies.

Design: Thirty-one renal biopsies with ATI were studied that included six patients with mild ATI (serum creatinine $(\mathrm{sCr})<2 \mathrm{mg} / \mathrm{dl}), 11$ with moderate ATI $(\mathrm{sCr} 2-4 \mathrm{mg} / \mathrm{dl}$ ) and 14 with severe ATI ( $\mathrm{sCr}$ at $>4 \mathrm{mg} / \mathrm{dl}$ ). The biopsies were stained for CD68 (cytoplasm) and KIM-1 (luminal cell membrane of proximal tubules) by immunohistochemistry with a stain intensity scoring on a scale of $0-3$. The patients' $\mathrm{SCr}$ levels on the biopsy day, within 1 month and within 6 month were obtained through chart review. Changes in $\mathrm{sCr}$ from the biopsy day minus 1-month or 6-month values were counted as recovery status (larger delta=better recovery).

Results: Control kidney tissue stained entirely negatively for both CD68 and KIM-1. CD68 was expressed in mild to moderate ATI, but was weakly expressed or negative in severely ATI. KIM-1 showed light staining in early ATI but expression became much stronger in severe injured ATI. All mild ATI cases had CD68/KIM-1 ratio $\geq 1$ and showed good recovery in $\mathrm{sCr}$ function over 1 to 6 months. Seven out of eleven (45\%) cases in moderate ATI and 6/14 (43\%) in severe ATI had CD68/KIM-1 ratio $>1$, respectively. Within 1 month, renal function recovery was not significantly associated with CD68 KIM-1 $(r=0.046, p=0.813)$. At 6 months of follow-up, the CD68/KIM-1 ratio was significantly associated with $\mathrm{SCr}$ recovery $(\mathrm{r}=0.459$ and $\mathrm{p}=0.0315)$.

Conclusions: Our data indicate that CD68/KIM-1 ratio $\geq 1$ in renal biopsies carries a useful value in predicting renal functional recovery at half a year; the higher the ratio the better $\mathrm{sCr}$ recovery (dual stains assuming repairing process but reversed CD68 KIM-1 ratio implying a loss of tubular repairing capacity).

1648 Increasing Tumor Burden in Radical Nephrectomy Specimens (RN) Corresponds to a Positive Delta in Estimated Glomerular Filtration Rate Over Time

Melanie Wooten, Muhammad Hassan, Thomas Rogers, Alton B Farris, Kenneth Ogan, Carla Ellis. Emory University, Atlanta, GA; Howard University, Washington, DC. Background: The effect of increasing tumor burden at RN on the estimated glomerular filtration rate (eGFR) over time and on the severity of non-neoplastic disease has yet to be studied in detail. Our study seeks to determine what if any clinicopathologic findings at $R N$ affect the change in eGFR ( $\triangle \mathrm{eGFR}$ ) from the time of surgery to the most recent patient follow up, and if the extent of malignancy has an effect on ipsilateral non-neoplastic disease.

Design: A search of our pathology system for all RN in 2009 was performed. Exclusion criteria included any patients without a contralateral kidney, that had undergone renal transplant, or with a clinical diagnosis of end stage renal disease. The remaining patients $(\mathrm{N}=47)$ were grouped by pathologic stage at $\mathrm{RN}$ : Group $1(\mathrm{~T} 1 \mathrm{a})$, group $2(\mathrm{~T} 1 \mathrm{~b})$, group 3 (T2), group 4 (T3a) and group 5 (T3b and above). $\triangle \mathrm{eGFR}$, defined as the eGFR (by CKD-EPI) at the time of surgery minus the eGFR at the time of last known follow up was recorded on all patients. In addition, the demographic information, comorbid factors and (when available) \% glomerulosclerosis, degree of interstitial fibrosis and tubular atrophy (IFTA) and degree of arteriosclerosis was evaluated for each patient. Mean follow up was 36 months.
Results: 47 total patients were evaluated. $60 \%$ were Caucasian and $53 \%$ were female Group 1 contained the highest percentage of females $(88 \%)$ as well as the highest percentage of comorbidity $(75 \%)$. The following factors were associated with a net positive $\triangle \mathrm{eGFR}$ : increasing $\mathrm{T}$ stage, aged between $41-50$ years and male gender. Caucasian ethnicity and evidence of the clear cell subtype of renal cell carcinoma (RCC) were associated with a net negative $\Delta$ eGFR, but improved over other ethnicities and RCC subtypes. \% glomerulosclerosis was highest in groups 3 and 4 (> than $10 \%$ ), IFTA increased in severity with increasing tumor burden and a history of hypertension was associated with at least mild arteriosclerosis.

Conclusions: Increasing tumor burden, male gender and relatively young age were associated with a net positive $\Delta \mathrm{eGFR}$ after RN. Increasing tumor burden may be related to increased time to develop compensatory mechanisms in the contralateral kidney; however it appears to be associated with increasing IFTA in the ipsilateral kidney, which emphasizes the importance of documentation of non-neoplastic disease at the time of RN.

\section{Convergence of Vasculopathy and Vasculitis: Digital Profiling} as a Novel Approach for the Study of the Renal Biopsy

Jia Xu, Milan Rosen, Christina I Luffman, Beverly E Faulkner-Jones, Seymour Rosen. BIDMC, Boston, MA.

Background: Scleroderma vasculopathy and ANCA-associated glomerulonephritis have been rarely reported to occur simultaneously in one patient. Herein, we report a patient who presented with a classic constellation of clinical and laboratory findings of systemic scleroderma, and was subsequently found to be positive for p-ANCA. Two renal biopsies, performed six months apart demonstrated typical changes of the two entities in both acute and "healed" phases.

Design: Two renal biopsies were serially sectioned and stained routinely, and with CD31 and CD34 as endothelial markers. The slides were digitized, aligned and analyzed. Each glomerular tuft was sequentially studied in terms of total area (um2) and each biopsy was individually profiled. All arterial vessels were sequentially studied with whole vessel and luminal areas delineated and ratios calculated.

Results: The initial biopsy contained 16 glomeruli all with extensive fibrinoid necrosis and destruction of the capillary network. The arterial vessels (interlobular and arcuate) showed intimal edema with luminal occlusion. CD31/CD34 showed variable endothelial intactness, but demonstrated the luminal size shifts. The second biopsy had 23 glomeruli that were either segmentally or globally sclerotic with no active changes. The vessels were now normally patent. Figure1 (first biopsy) shows a segmented profile of a CD34 stain with the glomerular areas (um2) calculated and the vascular lumina expressed as a percentage of the total vessel cross-sectional area. Figure 2 (Masson Trichrome, second biopsy) shows the same parameters. There is a marked diminishment in glomerular size with variability and a return to "normal" vascular lumina.

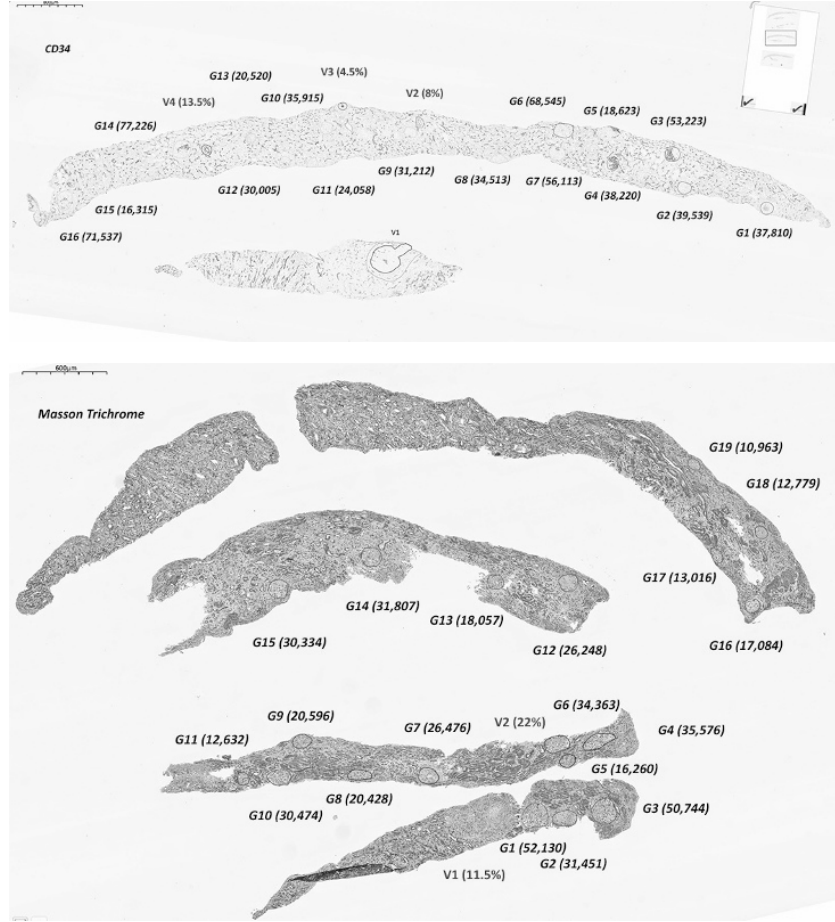

Conclusions: These studies depict the rare occurrence of two entities together, the scleroderma kidney vasculopathy and the glomerulonephritis of ANCA-associated vasculitis syndrome both in an acute and healing phase, profiled by computer mapping techniques.

1650 Pathology of Obstruction in Pediatric Renal Allografts, a Mechanism of Interstitial Fibrosis in Renal Transplantation

Chen Yang, Joseph Gaut. Washington University Medical Center, St. Louis, MO. Background: In the pediatric population, obstructive nephropathy $(\mathrm{ON})$ is a common indication for renal transplantation. However, pathologic disease recurrence in allografts has received little attention in the published literature. Patients with ON demonstrate 
segmental interstitial fibrosis (IF) without global glomerulosclerosis, a pattern often associated with chronic calcineurin inhibitor (CNI) toxicity. Patients with CNI toxicity also commonly demonstrate arteriolar hyalinosis. Distinguishing CNI toxicity from recurrent obstruction has implications for clinical management. The aim of this study is to investigate the pathologic findings in pediatric renal transplant recipients with a history of obstructive nephropathy.

Design: The institutional surgical pathology archives were searched for pediatric patients with and without a history of $\mathrm{ON}$ who underwent a kidney transplant biopsy between years 2010 and 2015. A total of 16 consecutive patients with a history of ON and 12 consecutive patients without a history of ON were identified. Biopsies were reviewed by two pathologists and scored using current Banff criteria. Clinical and radiologic data were collected and summarized.

Results: Of the 16 patients transplanted for ON, $9(56 \%)$ showed evidence of segmental IF without arteriolar hyalinosis or significant global glomerulosclerosis $(<10 \%)$. Of the cases with segmental IF, 6 showed obstructive changes on imaging; 4 showed neutrophilic tubulitis and white blood cell casts; 2 subsequently developed acute pyelonephritis. Of the 12 patients transplanted for other reasons, 3 (25\%) showed evidence of segmental IF without arteriolar hyalinosis or global glomerulosclerosis. Routine imaging studies were not performed for the majority of these cases. None developed pyelonephritis. There was no significant difference in blood CNI concentration between the two groups. None of the cases that showed segmental IF met the criteria for acute cellular or antibody mediated rejection.

Conclusions: Segmental IF, in the absence of arteriolar hyalinosis or glomerulosclerosis, is more common in patients with recurrent $\mathrm{ON}$ in renal allografts $(\mathrm{p}<0.05)$. These findings should not be assumed to be related to CNI toxicity. Observation of similar pathology in patients without a history of ON raises the possibility that obstruction may be contributing to chronic graft injury. Further investigations evaluating the role of obstruction as a contributing factor to graft loss are warranted.

1651 The Spectrum of Podocyte and Parietal Epithelial Cells (PEC) Activation in Nonsclerosed Glomeruli in Focal Segmental Sclerosis (FSGS) Haichun Yang, Miguel F Palma Diaz, Giovanna Giannico, Sheau-Chiann Chen, Sharon Philips, Charles Jennette, Arthur H Cohen, James M Pullman, Vivette D'Agati, Charles E Alpers, Agnes Fogo. Vanderbilt University Medical Center, Nashville, TN; UCLA David Geffen School of Medicine, Los Angeles, CA; University of North Carolina, Chapel Hill, NC; Wake Forest School of Medicine, Winston-Salem, NC; Montefiore Medical Center, Bronx, NY; Columbia University, New York, NY; University of Washington, Seattle, WA.

Background: Primary FSGS develops from podocyte injury followed by PEC activation. This process is associated with phenotypic changes detectable by immunohistochemistry in both cell types, and which may vary among the morphologic FSGS variants: collapsing, tip, cellular, perihilar, and not otherwise specified (NOS). Design: We assessed podocyte injury and PEC activation by immunostaining for WT-1 (differentiated podocytes), claudin (PECs) and CD44 (activated PECs) in 201 primary FSGS biopsies, including collapsing (44), tip (54), cellular (27), perihilar (22), and NOS (54) variants. Minimal change disease biopsies $(\mathrm{MCD}, \mathrm{n}=19)$ and normal nephrectomy cases $(\mathrm{n}=20)$ were used as controls. We compared intact vs sclerosed glomeruli, and nonlesional vs segmental lesional areas in the same glomerulus.

Results: Glomeruli from all FSGS variants had fewer WT-1 positive (+) cells and more claudin and CD44 + cells than control or MCD cases. Tip FSGS had the most WT-1 and the fewest claudin and CD44 + cells, while collapsing FSGS had the fewest WT-1 and the most claudin and CD44 + cells. Nonsclerosed glomeruli in all FSGS variants had fewer WT-1 + cells and more CD44 + cells compared to normal or MCD glomeruli. Segmentally sclerosed compared to nonsclerosed FSGS glomeruli showed even fewer WT- $1+$ cells and more claudin and CD44 + cells. The nonlesional areas of segmentally sclerosed glomeruli had similar numbers of WT-1, claudin and CD44 positive cells as nonsclerosed FSGS glomeruli, but still different from control or MCD.

Conclusions: Fewer WT-1 + and more claudin and CD44 + cells are detected in nonsclerosed FSGS glomeruli and intact areas of glomeruli with segmental lesions than in controls, suggesting podocyte injury and PEC activation may precede and cause the structural FSGS lesion. Differences in staining for the three markers between the least and most severe FSGS variants, tip and collapsing, further suggest that they predict severity of injury, and suggest different mechanisms among the FSGS variants.

\section{Post-Transplant Lymphoproliferative Disorder (PTLD) in Kidney} Transplant (KTx) Recipients

Pingchuan Zhang, Rebecca King, Kay M Ristow, Fernando Cosio, Lynn D Cornell, Thomas M Habermann, Mariam P Alexander. Mayo Clinic, Rochester, MN.

Background: Although PTLD is the second most common neoplasm in KTx patients, a diagnosis of PTLD is rarely rendered in the kidney biopsy (Kbx). This study focuses on PTLD in KTx recipients with a focus on renal PTLD emphasizing diagnostic challenges and outcomes.

Design: The clinical databases were searched for KTx-related PTLD from Jan 1970 to Aug 2016. The clinical and followup data were obtained. The cases with suspected kidney involvement were reviewed and re-classified (2016 WHO lymphoma classification).

Results: Seventy-seven KTx patients diagnosed with PTLD were identified. Table 1 shows their clinical and follow-up data. Of these, $10(13 \%)$ were suspected to have PTLD on Kbx. On review, 3 were excluded as reactive hyperplasia (EBV negative). Seven cases were involved by lymphoma (Table 2). A female predominance was observed. Monomorphic (B cell predominant) and polymorphic forms of PTLD were noted. Five were diagnosed with acute cellular rejection concurrently or 4 wks prior to PTLD diagnosis. EBV was positive in $60 \%$. Mean follow-up was 160 months; 6 patients survived PTLD following various treatment regimens:graft nephrectomy, immunosuppression reduction, Rituximab \& chemotherapy.
Conclusions: KTx recipients develop PTLD infrequently, with multi-organ involvement being common. Kidney PTLD is rare and differentiating it from rejection, with which it might coexist, is challenging. Use of ancillary tests allows appropriate treatment.

\begin{tabular}{|l|c|}
\hline Variables & Data \\
\hline $\mathrm{N}$ & 77 \\
\hline Age at Tx (yrs) & $49(21-72)$ \\
\hline Age at PTLD (yrs) & $56(21-84)$ \\
\hline M:F & $1.6: 1$ \\
\hline Time from Tx to PTLD (mth) & $78(1-500)$ \\
\hline Affected sites & \\
\hline Kidney & $13 \%$ \\
\hline Lymph node & $54 \%$ \\
\hline Liver & $21 \%$ \\
\hline GI tract & $25 \%$ \\
\hline Lung & $12 \%$ \\
\hline CNS & $11 \%$ \\
\hline Multiple organs & $67 \%$ \\
\hline Extra-nodal & $87 \%$ \\
\hline EBV positive & $62 \%$ \\
\hline CD20 positive & $68 \%$ \\
\hline Death & $41 \%$ \\
\hline
\end{tabular}

\begin{tabular}{|l|l|l|l|l|l|l|}
\hline Case & $\begin{array}{l}\text { Age at Dx } \\
\text { /sex }\end{array}$ & $\begin{array}{l}\text { Time (mo) } \\
\text { after Tx }\end{array}$ & $\begin{array}{l}\text { PTLD/ } \\
\text { lymphoma }\end{array}$ & $\begin{array}{l}\text { Cellular } \\
\text { rejection }\end{array}$ & $\begin{array}{l}\text { EBV } \\
\text { ISH }\end{array}$ & $\begin{array}{l}\text { Follow-up } \\
\text { (mo) }\end{array}$ \\
\hline 1 & $31 / \mathrm{F}$ & 54 & monomorphic & Yes & ND & A (72) \\
\hline 2 & $42 / \mathrm{F}$ & 72 & polymorphic & No & rare & U \\
\hline 3 & $46 / \mathrm{F}$ & 55 & polymorphic & Yes & neg & U \\
\hline 4 & $75 / \mathrm{M}$ & 60 & Lymphoplasmacytic* & No & neg & $\mathrm{U}$ \\
\hline 5 & $59 / \mathrm{F}$ & 16 & monomorphic & Yes & pos & $\mathrm{A}(66)$ \\
\hline 6 & $51 / \mathrm{F}$ & 38 & polymorphic & Yes & pos & $\mathrm{A}(22)$ \\
\hline 7 & $50 / \mathrm{M}$ & 58 & monomorphic T & Yes & ND & $\mathrm{D}(1)$ \\
\hline
\end{tabular}

\section{Immune Complex Kidney Disease and HIV-Associated} Nephropathy in Renal Allografts of HIV Infected Patients

Shuling Zheng, Stephen C Ward, Kenneth T Hughes, Fadi Salem. Icahn School of Medicine at Mount Sinai, New York, NY.

Background: HIV associated nephropathy (HIVAN) and HIV immune complex kidney disease (HIVICK) are the two most common HIV-related kidney diseases. HIVICK encompasses a group of entities with immune deposits identified on immunostaining and/or electron microscopy and may result from dysregulated immune response to HIV viruses or coinfections. As treatments for HIV have improved, more HIV patients are undergoing kidney transplantation. However, little is known about the development of immune complex kidney disease (ICKD) and HIVAN in the post-transplant setting. Design: Medical records were retrospectively reviewed from HIV positive patients who underwent renal transplantation and subsequent allograft biopsies performed at least one month after transplantation (2003-2015). All allografts were from HIV negative standard criteria donors.

Results: Biopsy reports were available for 60 allografts from 58 patients $(2$ patients received 2 allografts). Of these, 33 allografts were biopsied only within the first year post-transplantation (YPT), while the remaining 27 allografts were biopsied up to 9 YPT. Overall, 4 HIVAN and 6 ICKD cases were identified (1 patient had both). The incidence of HIVAN was $7 \%$. ICKD cases included 2 IgA nephropathy (IgAN), 3 membranous nephropathy (MN) and 1 not otherwise specified (who also had HIVAN). Three HIVAN and 1 IgAN cases were diagnosed $<1$ YPT. In allografts with biopsies $>1$ YPT, the incidence of ICKD was $19 \%(5 / 27)$. Anti-PLA2R antibodies (Abs) were detected in $1 \mathrm{MN}$ case. The HCV co-infection rate was $2 / 4(50 \%), 2 / 5(40 \%)$ and $16 / 49(33 \%)$ in HIVAN, ICKD and the remaining patients, respectively. The mean CD4 count (cells/ $\mathrm{ml})$ was $548(439-692 ; \mathrm{n}=3), 540(116-979 ; \mathrm{n}=5)$ and $292(34-956 ; \mathrm{n}=42)$ in HIVAN, ICKD and the remaining patients, respectively. The HIV viral load $(\mathrm{cp} / \mathrm{ml})$ was 111 and 170 in 2 HIVAN patients and 90 and 321 in 2 ICKD patients. The mean HIV viral load was $139 \mathrm{cp} / \mathrm{ml}(20-538 ; \mathrm{n}=18)$ in the remaining cohort. There was no significant difference in HCV co-infection rate, CD4 count or HIV viral load among the groups. Conclusions: In this cohort study, HIVAN occurred in $7 \%$ of allografts in HIV positive patients while ICKD occurred in 19\% of allografts with at least 1 year follow-up. The MN case with Anti-PLA2R Abs is most likely idiopathic, but the remaining ICKD cases may represent HIVICK in the post-transplant setting. More studies are needed to further elucidate the role of HIVICK and HIVAN in HIV positive post-transplant patients. 